\title{
Periodic Solutions for Shunting Inhibitory Cellular Neural Networks of Neutral Type with Time-Varying Delays in the Leakage Term on Time Scales
}

\author{
Yongkun Li, ${ }^{1}$ Lei Wang, ${ }^{1}$ and Yu Fei ${ }^{2}$ \\ ${ }^{1}$ Department of Mathematics, Yunnan University, Kunming, Yunnan 650221, China \\ ${ }^{2}$ School of Statistics and Mathematics, Yunnan University of Finance and Economics, \\ Kunming, Yunnan 650221, China
}

Correspondence should be addressed to Yongkun Li; yklie@ynu.edu.cn

Received 11 September 2013; Accepted 25 November 2013; Published 9 February 2014

Academic Editor: Yansheng Liu

Copyright @ 2014 Yongkun Li et al. This is an open access article distributed under the Creative Commons Attribution License, which permits unrestricted use, distribution, and reproduction in any medium, provided the original work is properly cited.

\begin{abstract}
A class of shunting inhibitory cellular neural networks of neutral type with time-varying delays in the leakage term on time scales is proposed. Based on the exponential dichotomy of linear dynamic equations on time scales, fixed point theorems, and calculus on time scales we obtain some sufficient conditions for the existence and global exponential stability of periodic solutions for that class of neural networks. The results of this paper are completely new and complementary to the previously known results even if the time scale $\mathbb{T}=\mathbb{R}$ or $\mathbb{Z}$. Moreover, we present illustrative numerical examples to show the feasibility of our results.
\end{abstract}

\section{Introduction}

As we know, shunting inhibitory cellular neural networks (SCINNs) have been applied in a wide range of practical fields such as psychophysics, speech, perception, robotics, adaptive pattern recognition, and image processing. Hence, they have been the object of intensive analysis by numerous authors in recent years. In particular, there have been extensive results on the problem of the existence and stability of periodic solutions and almost periodic solutions for SCINNs in the literature. For example, in [1-3], authors consider the existence and stability of almost periodic solutions for SCINNs; in $[4,5]$, authors consider the existence and stability of periodic solutions for SCINNs; in [5], authors by using the continuation theorem of coincidence degree theory and constructing suitable Lyapunov functions consider the periodic solution for SCINNs; in [6,7], authors obtained some sufficient conditions for the existence and stability of an equilibrium point.

Recently, another type of time delays, namely, neutral type time delays, which always appears in the study of automatic control, population dynamics, and vibrating masses attached to an elastic bar, and so forth, has recently drawn much research attention. There are some results on the stability and the existence of periodic solutions to delayed neural networks of neutral type, for example in [8-13], by using the Lyapunov functions and the linear matrix inequality approach, authors studied the asymptotic stability or exponential stability of the equilibrium point for delayed neural networks of neutral type and in $[14,15]$, by using the theory of abstract continuation theorem of $k$-set contractive operator, authors studied the existence of periodic solutions for delayed cellular neural networks of neutral type and Hopfield neural networks with neutral delays, respectively. In a recent paper [16], authors studied the existence and exponential stability for the following SICNN with continuously distributed delays of neutral type:

$$
\begin{aligned}
x_{i j}^{\prime}(t)= & -a_{i j}(t) x_{i j}(t)-\sum_{C_{k l} \in N_{r}(i, j)} C_{i j}^{k l}(t) \\
& \times \int_{0}^{+\infty} K_{i j}(u) f\left[x_{k l}(t-u)\right] d u x_{i j}(t)
\end{aligned}
$$




$$
\begin{aligned}
& -\sum_{C_{k l} \in N_{s}(i, j)} D_{i j}^{k l}(t) \\
& \times \int_{0}^{+\infty} J_{i j}(u) g\left[x_{k l}^{\prime}(t-u)\right] d u x_{i j}(t)+I_{i j}(t),
\end{aligned}
$$

where $C_{i j}$ is the cell at the $(i, j)$ position of the lattice, the $r$ neighborhood $N_{r}(i, j)$ of $C_{i j}$ is defined as follows:

$$
\begin{gathered}
N_{r}(i, j)=\left\{C_{k l}: \max \{|k-i|,|l-j|\} \leq r,\right. \\
1 \leq k \leq m, 1 \leq l \leq n\},
\end{gathered}
$$

where $x_{i j}$ represents the state of the cell $C_{i j}$, the coefficient $a_{i j}(t)>0$ means the passive decay rate of the passive decay rate of the cell activity, and $C_{i j}^{k l}(t)$ and $D_{i j}^{k l}(t)$ describe the connection or coupling strength of postsynaptic activity of the cell $C_{k l}$ transmitted to the cell $C_{i j}$.

Very recently, a leakage delay, which is the time delay in the leakage term of the systems and a considerable factor affecting dynamics for the worse in the systems, is being put to use in the problem of stability for neural networks $[17,18]$. However, so far, very little attention has been paid to neural networks with time delay in the leakage (or "forgetting") term [19-25]. Such time delays in the leakage term are difficult to handle but have great impact on the dynamical behavior of neural networks.

Also, it is well known that both continuous time and discrete time neural networks have equal importance in various applications. Moreover, the theory of calculus on time scales was initiated by Stefan Hilger [26] in his Ph.D. thesis in order to unify continuous and discrete analysis, and it has a tremendous potential for applications and has recently received much attention since his foundational work. For instance, in [27], the authors studied antiperiodic solutions to impulsive SICNNs with distributed delays on time scales. In [28], the authors studied almost periodic solutions of SCINNs on time scales. However, to the best of our knowledge, there is no paper published on the existence and stability of periodic solutions for SCINNs of neutral type with the time delay in the leakage term.

Motivated by the above discussions, in this paper, we are concerned with the following SCINNs of neutral type with time-varying delays in the leakage term on time scale $\mathbb{T}$ :

$$
\begin{aligned}
x_{i j}^{\Delta}(t)= & -a_{i j}(t) x_{i j}\left(t-\mu_{i j}(t)\right)-\sum_{C_{k l} \in N_{r}(i, j)} C_{i j}^{k l}(t) \\
& \times \int_{0}^{+\infty} K_{i j}(u) f\left[x_{k l}(t-u)\right] \Delta u x_{i j}(t) \\
& -\sum_{C_{k l} \in N_{s}(i, j)} D_{i j}^{k l}(t) \\
& \times \int_{0}^{+\infty} J_{i j}(u) g\left[x_{k l}^{\Delta}(t-u)\right] \Delta u x_{i j}(t)+I_{i j}(t), \\
&
\end{aligned}
$$

where $\mathbb{T}$ is a periodic time scale and $\mathbb{T}^{+}=\mathbb{T} \cap[0,+\infty), C_{i j}$ is the cell at the $(i, j)$ position of the lattice, the $r$ neighborhood $N_{r}(i, j)$ of $C_{i j}$ is defined as follows:

$$
\begin{gathered}
N_{r}(i, j)=\left\{C_{k l}: \max \{|k-i|,|l-j|\} \leq r,\right. \\
1 \leq k \leq m, 1 \leq l \leq n\}
\end{gathered}
$$

$x_{i j}$ represents the state of the cell $C_{i j}$, the coefficient $a_{i j}(t)>$ 0 means the passive decay rate of the passive decay rate of the cell activity, and $C_{i j}^{k l}(t)$ and $D_{i j}^{k l}(t)$ describe the connection or coupling strength of postsynaptic activity of the cell $C_{k l}$ transmitted to the cell $C_{i j}$. follows:

The initial condition associated with (3) is defined as

$$
x_{i j}(s)=\psi_{i j}(s), \quad s \in(-\infty, 0]_{\mathbb{T}},
$$

where $i=1,2, \ldots, n, j=1,2, \ldots, m$, and $\psi_{i j}(s)$ are $\Delta$ differentiable.

Clearly, if $\mathbb{T}=\mathbb{R}$ and $\mu_{i j} \equiv 0$, then (3) reduces to (1).

Our main purpose of this paper is to study the existence and global exponential stability of periodic solutions to (3) by using the exponential dichotomy of linear dynamic equations on time scales and some inequality technics. Our results of this paper are completely new and complementary to the previously known results even if the time scale $\mathbb{T}=\mathbb{R}$ or $\mathbb{Z}$. Our methods used in this paper are different from those used in $[14,15,19]$ and can be used to study other types' delayed neural networks of neutral type with delays in the leakage term.

For convenience, we denote $[a, b]_{\mathbb{T}}:=\{t \mid t \in[a, b] \cap \mathbb{T}\}$. And we introduce the following notations:

$$
\begin{array}{rr}
a_{i j}^{M}=\sup _{t \in \mathbb{R}}\left|a_{i j}(t)\right|, & a_{i j}^{m}=\inf _{t \in \mathbb{R}}\left|a_{i j}(t)\right|, \\
\mu_{i j}^{M}=\sup _{t \in \mathbb{R}}\left|\mu_{i j}(t)\right|, \quad \overline{C_{i j}^{k l}}=\sup _{t \in \mathbb{R}}\left|C_{i j}^{k l}(t)\right|, \\
\overline{D_{i j}^{k l}}=\sup _{t \in \mathbb{R}}\left|D_{i j}^{k l}(t)\right|, \quad \overline{I_{i j}}=\sup _{t \in \mathbb{R}}\left|I_{i j}(t)\right|, \\
i=1,2, \ldots, n, \quad j=1,2, \ldots, m .
\end{array}
$$

Throughout this paper, we assume that the following conditions hold:

$$
\begin{aligned}
\left(H_{1}\right) & C_{i j}^{k l}(t), D_{i j}^{k l}(t), I_{i j}(t) \in C(\mathbb{T}, \mathbb{R}), a_{i j}(t) \in C\left(\mathbb{T}, \mathbb{R}^{+}\right), \text {and } \\
& \mu_{i j}(t) \in C\left(\mathbb{T}, \mathbb{T}^{+}\right) \text {are all } \omega \text {-periodic functions, for } u \in \\
& (0,+\infty)_{\mathbb{T}} \text { and } t \in \mathbb{T}, t-u \in \mathbb{T} \text { and for } t \in \mathbb{T}, t-\mu_{i j}(t) \in \\
& \mathbb{T}, i=1,2, \ldots, n, j=1,2, \ldots, m .
\end{aligned}
$$

$\left(H_{2}\right) f_{j}, g_{j} \in C(\mathbb{R}, \mathbb{R})$ and there exist positive constants $L, l$ such that

$$
\begin{gathered}
\left|f_{j}(u)-f_{j}(v)\right| \leq L|u-v|, \\
\left|g_{j}(u)-g_{j}(v)\right| \leq l|u-v|,
\end{gathered}
$$

for all $u, v \in \mathbb{R}, j=1,2, \ldots, m$. 
$\left(H_{3}\right)$ For $i=1,2, \ldots, n, j=1,2, \ldots, m$, the delay kernels $K_{i j}, J_{i j}:[0, \infty) \cap \mathbb{T} \rightarrow \mathbb{R}$ are continuous and integrable with

$$
0 \leq \int_{0}^{\infty}\left|K_{i j}(s)\right| \Delta s \leq K_{i j}^{0}, \quad 0 \leq \int_{0}^{\infty}\left|J_{i j}(s)\right| \Delta s \leq J_{i j}^{0}
$$

This paper is organized as follows: in Section 2, we introduce some notations and definitions and state some preliminary results which are needed in later sections. In Section 3, we establish some sufficient conditions for the existence of periodic solutions of (3). In Section 4, we prove that the periodic solution obtained in Section 3 is globally exponentially stable. In Section 5, we give examples to illustrate the feasibility of our results obtained in previous sections.

\section{Preliminaries}

In this section, we introduce some definitions and state some preliminary results.

Definition 1 (see [29]). Let $\mathbb{T}$ be a nonempty closed subset (time scale) of $\mathbb{R}$. The forward and backward jump operators $\sigma, \rho: \mathbb{T} \rightarrow \mathbb{T}$ and the graininess $\mu: \mathbb{T} \rightarrow \mathbb{R}^{+}$are defined, respectively, by

$$
\begin{gathered}
\sigma(t)=\inf \{s \in \mathbb{T}: s>t\}, \\
\rho(t)=\sup \{s \in \mathbb{T}: s<t\}, \quad \mu(t)=\sigma(t)-t .
\end{gathered}
$$

Definition 2 (see [29]). A point $t \in \mathbb{T}$ is called left-dense if $t>$ inf $\mathbb{T}$ and $\rho(t)=t$, left-scattered if $\rho(t)<t$, right-dense if $t<\sup \mathbb{V}$ and $\sigma(t)=t$, and right-scattered if $\sigma(t)>t$. If $\mathbb{T}$ has a left-scattered maximum $m$, then $\mathbb{T}^{k}=\mathbb{T} \backslash\{m\}$; otherwise $\mathbb{T}^{k}=\mathbb{T}$. If $\mathbb{T}$ has a right-scattered minimum $m$, then $\mathbb{T}^{k}=$ $\mathbb{T} \backslash\{m\}$; otherwise $\mathbb{T}^{k}=\mathbb{T}$.

Definition 3 (see [30]). One says that a time scale $\mathbb{T}$ is periodic if there exists $p>0$ such that if $t \in \mathbb{T}$, then $t \pm p \in \mathbb{T}$. For $\mathbb{T} \neq \mathbb{R}$, the smallest positive $p$ is called the period of the time scale.

Throughout this paper, we restrict our discussions in periodic time scales.

Definition 4 (see [29]). A function $r: \mathbb{T} \rightarrow \mathbb{R}$ is called regressive if

$$
1+\mu(t) r(t) \neq 0
$$

for all $t \in \mathbb{T}^{k}$. The set of all regressive and $\mathrm{rd}$-continuous functions $r: \mathbb{T} \rightarrow \mathbb{R}$ will be denoted by $\mathscr{R}=\mathscr{R}(\mathbb{\mathbb { T }})=$ $\mathscr{R}(\mathbb{T}, \mathbb{R})$; one defines the set $\mathscr{R}^{+}=\mathscr{R}^{+}(\mathbb{T}, \mathbb{R})=\{r \in \mathscr{R}, 1+$ $\mu(t) r(t)>0, \forall t \in \mathbb{T}\}$. If $r$ is regressive function, then the generalized exponential function $e_{r}$ is defined by

$$
e_{r}(t, s)=\exp \left\{\int_{s}^{t} \xi_{\mu(\tau)}(r(\tau)) \Delta \tau\right\}, \quad \text { for } s, t \in \mathbb{T},
$$

with the cylinder transformation

$$
\xi_{h}(z)= \begin{cases}\frac{\log (1+h z)}{h} & \text { if } h \neq 0 \\ z & \text { if } h=0\end{cases}
$$

Let $p, q: \mathbb{T} \rightarrow \mathbb{R}$ be two regressive functions, we define

$$
\begin{gathered}
p \oplus q:=p+q+\mu p q, \quad \quad \ominus p:=-\frac{p}{1+\mu p}, \\
p \ominus q:=p \oplus(\ominus q) .
\end{gathered}
$$

Then the generalized exponential function has the following properties.

Lemma 5 (see [29]). Assume that $p, q: \mathbb{T} \rightarrow \mathbb{R}$ are two regressive functions, then

(i) $e_{0}(t, s) \equiv 1$ and $e_{p}(t, t) \equiv 1$;

(ii) $e_{p}(\sigma(t), s)=(1+\mu(t) p(t)) e_{p}(t, s)$;

(iii) $e_{p}(t, s)=\left(1 / e_{p}(s, t)\right)=e_{\ominus p}(s, t)$;

(iv) $e_{p}(t, s) e_{p}(s, r)=e_{p}(t, r)$;

(v) $e_{p}(t, s) e_{q}(t, s)=e_{p \oplus q}(t, s)$.

Lemma 6 (see [29]). Assume that $f, g: \mathbb{T} \rightarrow \mathbb{R}$ are delta differentiable at $t \in \mathbb{T}^{k}$, then

(i) $\left(\nu_{1} f+v_{2} g\right)^{\Delta}=v_{1} f^{\Delta}+v_{2} g^{\Delta}$ for any constants $\nu_{1}, v_{2}$;

(ii) $(f g)^{\Delta}(t)=f^{\Delta}(t) g(t)+f(\sigma(t)) g^{\Delta}(t)=f(t) g^{\Delta}(t)+$ $f^{\Delta}(t) g(\sigma(t))$

(iii) if $f^{\Delta} \geq 0$, then $f$ is nondecreasing.

Lemma 7 (see [29]). Assume that $p(t) \geq 0$ for $t \geq s$, then $e_{p}(t, s) \geq 1$.

Lemma 8 (see [29]). Suppose that $p \in \mathscr{R}^{+}$, then

(i) $e_{p}(t, s)>0$, for all $t, s \in \mathbb{T}$;

(ii) if $p(t) \leq q(t)$ for all $t \geq s, t, s \in \mathbb{T}$, then $e_{p}(t, s) \leq$ $e_{q}(t, s)$ for all $t \geq s$.

Definition 9 (see [29]). If $a \in \mathbb{T}$, sup $\mathbb{T}=\infty$ and $f$ is rdcontinuous on $[a, \infty)$, then one defines the improper integral by

$$
\int_{a}^{\infty} f(t) \Delta t=\lim _{b \rightarrow \infty} \int_{a}^{b} f(t) \Delta t
$$

provided this limit exists, and one says that the improper integral converges in this case. If this limit does not exist, then one says that the improper integral diverges.

Definition 10 (see [29]). If $a \in \mathbb{T}$, inf $\mathbb{T}=-\infty$, and $f$ is rd-continuous on $(-\infty, a)$, then one defines the improper integral by

$$
\int_{-\infty}^{a} f(t) \Delta t=\lim _{b \rightarrow-\infty} \int_{b}^{a} f(t) \Delta t
$$


provided this limit exists, and we say that the improper integral converges in this case. If this limit does not exist, then one says that the improper integral diverges.

Lemma 11 (see [29]). Let $a \in \mathbb{T}^{k}$ and $b \in \mathbb{T}$ and assume that $f: \mathbb{T} \times \mathbb{T}^{k} \rightarrow \mathbb{R}$ is continuous at $(t, t)$, where $t \in \mathbb{T}^{k}$ with $t>a$. Also assume that $f^{\Delta}(t, \cdot)$ is $r d$-continuous on $[a, \sigma(t)]$. Suppose that for each $\varepsilon>0$, there exists a neighborhood $U$ of $\tau \in[a, \sigma(t)]$ such that

$$
\begin{aligned}
& \left|f(\sigma(t), \tau)-f(s, \tau)-f^{\Delta}(t, \tau)(\sigma(t)-s)\right| \\
& \quad \leq \varepsilon|\sigma(t)-s|, \quad \forall s \in U,
\end{aligned}
$$

where $f^{\Delta}$ denotes the derivative of $f$ with respect to the first variable. Then

(i) $g(t):=\int_{a}^{t} f(t, \tau) \Delta \tau$ implies $g^{\Delta}(t):=\int_{a}^{t} f^{\Delta}(t, \tau) \Delta \tau+$ $f(\sigma(t), t)$;

(ii) $h(t):=\int_{t}^{b} f(t, \tau) \Delta \tau$ implies $h^{\Delta}(t):=\int_{a}^{t} f^{\Delta}(t, \tau) \Delta \tau-$ $f(\sigma(t), t)$.

Definition 12 (see [31]). Let $X \in \mathbb{R}^{n}$ and $A(t)$ be a $n \times n$ matrix-valued function on $\mathbb{T}$, the linear system

$$
X^{\Delta}(t)=A(t) X(t), \quad t \in \mathbb{T},
$$

is said to admit an exponential dichotomy on $\mathbb{T}$ if there exist positive constants $k_{i}$ and $\alpha_{i}, i=1,2$, projection $P$, and the fundamental solution matrix $X(t)$ of (17) satisfying

$$
\begin{array}{r}
\left|X(t) P X^{-1}(\sigma(s))\right| \leq k_{1} e_{\ominus \alpha_{1}}(t, s), \quad s, t \in \mathbb{T}, t \geq s, \\
\left|X(t)(I-P) X^{-1}(\sigma(s))\right| \leq k_{2} e_{\ominus \alpha_{2}}(s, t), \quad s, t \in \mathbb{T}, t \leq s,
\end{array}
$$

where $|\cdot|$ is a matrix norm on $\mathbb{T}$; that is, if $A=\left(a_{i j}\right)_{n \times m}$, then we can take $|A|=\left(\sum_{i=1}^{n} \sum_{j=1}^{m}\left|a_{i j}\right|^{2}\right)^{1 / 2}$.

Lemma 13 (see [31]). If (17) admits an exponential dichotomy, then the following $\omega$-periodic system:

$$
x^{\Delta}(t)=A(t) x(t)+g(t), \quad t \in \mathbb{T},
$$

has an $\omega$-periodic solution as follows:

$$
\begin{aligned}
x(t)= & \int_{-\infty}^{t} X(t) P X^{-1}(\sigma(s)) g(s) \Delta s \\
& -\int_{t}^{+\infty} X(t)(I-P) X^{-1}(\sigma(s)) g(s) \Delta s,
\end{aligned}
$$

where $X(t)$ is the fundamental solution matrix of (17).

Lemma 14 (see [31]). If $A(t)$ is a uniformly bounded $r d$ continuous $n \times n$ matrix-valued function on $\mathbb{T}$ and there is a $\delta>0$ such that

$$
\begin{array}{r}
\left|a_{i i}(t)\right|-\sum_{j \neq i}\left|a_{i j}(t)\right|-\frac{1}{2} \mu(t)\left(\sum_{j=1}^{n}\left|a_{i j}(t)\right|\right)^{2} \\
-\delta^{2} \mu(t) \geq 2 \delta, \quad t \in \mathbb{T}, \quad i=1,2, \ldots, n,
\end{array}
$$

then (17) admits an exponential dichotomy on $\mathbb{T}$.
Lemma 15 (see [29]). If $p \in \mathscr{R}$ and $a, b, c \in \mathbb{T}$, then

$$
\begin{gathered}
{\left[e_{p}(c, \cdot)\right]^{\Delta}=-p\left[e_{p}(c, \cdot)\right]^{\sigma}} \\
\int_{a}^{b} p(t) e_{p}(c, \sigma(t)) \Delta t=e_{p}(c, a)-e_{p}(c, b) .
\end{gathered}
$$

\section{Existence of Periodic Solutions}

In this section, we will state and prove the sufficient conditions for the existence of periodic solutions of (3).

Set $\mathbb{X}=\left\{\varphi=\left\{\varphi_{i j}\right\} \quad \mid \varphi_{i j} \in C^{1}(\mathbb{T}, \mathbb{R}), \varphi\right.$ is an $\omega$ periodic function on $\mathbb{T}, i=1,2, \ldots, n, j=1,2, \ldots, m\}$ with the norm $\|\varphi\|=\max \left\{|\varphi|_{0},\left|\varphi^{\Delta}\right|_{0}\right\}$, where $|\varphi|_{0}=$ $\max _{i, j} \max _{t \in[0, \omega]_{\mathbb{T}}}\left|\varphi_{i j}(t)\right|,\left|\varphi^{\Delta}\right|_{0}=\max _{i, j} \max _{t \in[0, \omega]_{\mathbb{T}}}\left|\varphi_{i j}^{\Delta}(t)\right|$, $C^{1}(\mathbb{T}, \mathbb{R})$ is the set of continuous functions with continuous $\Delta$-derivatives on $\mathbb{T}$; then $\mathbb{X}$ is a Banach space. Let $\varphi^{0}(t)=\left\{\varphi_{i j}^{0}(t)\right\}$, where $\varphi_{i j}^{0}(t)=\int_{-\infty}^{t} e_{-a_{i j}}(t, \sigma(s)) I_{i j}(s) \Delta s, i=$ $1,2, \ldots, n, j=1,2, \ldots, m$ and let $A$ be a constant satisfying $A \geq \max \left\{\left\|\varphi^{0}\right\|, \max _{1 \leq j \leq n}\left|f_{j}(0)\right|, \max _{1 \leq j \leq n}\left|g_{j}(0)\right|\right\}$.

Theorem 16. Let $\left(H_{1}\right)-\left(H_{3}\right)$ hold. Suppose that

$\left(H_{4}\right)$ there exists a positive constant $\delta$ such that

$$
\begin{array}{r}
a_{i j}(t)-\frac{1}{2} \mu(t) a_{i j}^{2}(t)-\delta^{2} \mu(t) \geq 2 \delta, \\
t \in \mathbb{T}, \quad i=1,2, \ldots, n, \quad j=1,2, \ldots, m
\end{array}
$$

$\left(\mathrm{H}_{5}\right)$ more over

$$
\begin{aligned}
& \max _{\substack{1 \leq i \leq n \\
1 \leq j \leq m}}\left\{\frac{\theta_{i j}^{0}}{a_{i j}^{m}},\left(1+\frac{a_{i j}^{M}}{a_{i j}^{m}}\right) \theta_{i j}^{0}\right\} \leq \frac{1}{2}, \\
& \max _{\substack{1 \leq i \leq n \\
1 \leq j \leq m}}\left\{\frac{\theta_{i j}}{a_{i j}^{m}},\left(1+\frac{a_{i j}^{M}}{a_{i j}^{m}}\right) \theta_{i j}\right\}<1,
\end{aligned}
$$

where $i=1,2, \ldots, n, j=1,2, \ldots, m$,

$$
\begin{aligned}
\theta_{i j}^{0}= & a_{i j}^{M} \mu_{i j}^{M}+\sum_{C_{k l} \in N_{r}(i, j)} \overline{C_{i j}^{k l}} K_{i j}^{0}(2 A L+|f(0)|) \\
& +\sum_{C_{k l} \in N_{s}(i, j)} \overline{D_{i j}^{k l}} J_{i j}^{0}(2 A l+|g(0)|), \\
\theta_{i j}= & a_{i j}^{M} \mu_{i j}^{M}+\sum_{C_{k l} \in N_{r}(i, j)} \overline{C_{i j}^{k l}} K_{i j}^{0}(4 A L+|f(0)|) \\
& +\sum_{C_{k l} \in N_{s}(i, j)} \overline{D_{i j}^{k l}} J_{i j}^{0}(4 A l+|g(0)|) .
\end{aligned}
$$

Then (3) has a unique periodic solution in $\mathbb{X}_{0}=\{\varphi \in \mathbb{X} \mid$ $\left.\left\|\varphi-\varphi^{0}\right\| \leq A\right\}$. 
Proof. Rewrite (3) in the form

$$
\begin{aligned}
x_{i j}^{\Delta}(t)= & -a_{i j}(t) x_{i j}(t)+a_{i j}(t) \int_{t-\mu_{i j}(t)}^{t} x_{i j}^{\Delta}(s) \Delta s \\
& -\sum_{C_{k l} \in N_{r}(i, j)} C_{i j}^{k l}(t) \\
& \times \int_{0}^{+\infty} K_{i j}(u) f\left[x_{k l}(t-u)\right] \Delta u x_{i j}(t) \\
& -\sum_{C_{k l} \in N_{s}(i, j)} D_{i j}^{k l}(t) \\
& \times \int_{0}^{+\infty} J_{i j}(u) g\left[x_{k l}^{\Delta}(t-u)\right] \Delta u x_{i j}(t)+I_{i j}(t),
\end{aligned}
$$

where $i=1,2, \ldots, n, j=1,2, \ldots, m$. The initial condition associated with (3) is defined as follows:

$$
x_{i j}(s)=\psi_{i j}(s), \quad s \in(-\infty, 0]_{\mathbb{T}},
$$

where $i=1,2, \ldots, n, j=1,2, \ldots, m$, and $\psi_{i j}(s)$ are $\Delta$ differentiable. For any given $\varphi \in \mathbb{X}$, we consider the following periodic system:

$$
\begin{aligned}
& x_{i j}^{\Delta}(t)=-a_{i j}(t) x_{i j}(t)+a_{i j}(t) \int_{t-\mu_{i j}(t)}^{t} \varphi_{i j}^{\Delta}(s) \Delta s \\
&-\sum_{C_{k l} \in N_{r}(i, j)} C_{i j}^{k l}(t) \\
& \times \int_{0}^{+\infty} K_{i j}(u) f\left[\varphi_{k l}(t-u)\right] \Delta u \varphi_{i j}(t) \\
&-\sum_{C_{k l} \in N_{s}(i, j)} D_{i j}^{k l}(t) \\
& \times \int_{0}^{+\infty} J_{i j}(u) g\left[\varphi_{k l}^{\Delta}(t-u)\right] \Delta u \varphi_{i j}(t)+I_{i j}(t) \\
&=-a_{i j}(t) x_{i j}(t)+F_{i j}^{\varphi}(t)+I_{i j}(t), \\
& i=1,2, \ldots, n, j=1,2, \ldots, m,
\end{aligned}
$$

where

$$
\begin{aligned}
F_{i j}^{\varphi}(t)= & a_{i j}(t) \int_{t-\mu_{i j}(t)}^{t} \varphi_{i j}^{\Delta}(s) \Delta s \\
& -\sum_{C_{k l} \in N_{r}(i, j)} C_{i j}^{k l}(t) \\
& \times \int_{0}^{+\infty} K_{i j}(u) f\left[\varphi_{k l}(t-u)\right] \Delta u \varphi_{i j}(t) \\
& -\sum_{C_{k l} \in N_{s}(i, j)} D_{i j}^{k l}(t) \\
& \times \int_{0}^{+\infty} J_{i j}(u) g\left[\varphi_{k l}^{\Delta}(t-u)\right] \Delta u \varphi_{i j}(t), \\
& i=1,2, \ldots, n, \quad j=1,2, \ldots, m .
\end{aligned}
$$

Since $\left(H_{4}\right)$ holds, it follows from Lemma 14 that the linear system

$$
x_{i j}^{\Delta}(t)=-a_{i j}(t) x_{i j}(t), \quad i=1,2, \ldots, n, j=1,2, \ldots, m,
$$

admits an exponential dichotomy on $\mathbb{T}$. Thus, by Lemma 13, we obtain that (28) has an $\omega$-periodic solution, which is expressed as follows:

$$
\begin{array}{r}
x_{i j}^{\varphi}(t)=\int_{-\infty}^{t} e_{-a_{i j}}(t, \sigma(s))\left(F_{i j}^{\varphi}(s)+I_{i j}(s)\right) \Delta s, \\
i=1,2, \ldots, n, \quad j=1,2, \ldots, m .
\end{array}
$$

For $\varphi \in \mathbb{X}_{0}$, then $\|\varphi\| \leq\left\|\varphi-\varphi_{0}\right\|+\left\|\varphi_{0}\right\| \leq 2 A$. Define the following nonlinear operator:

$$
\Phi: \mathbb{X}_{0} \longrightarrow \mathbb{X}_{0}, \quad \varphi=\left\{\varphi_{i j}\right\} \longrightarrow x^{\varphi}=\left\{x_{i j}^{\varphi}\right\} .
$$

First we show that, for any $\varphi \in \mathbb{X}_{0}, \Phi(\varphi) \in \mathbb{X}_{0}$. Note that

$$
\begin{aligned}
& \left|F_{i j}^{\varphi}(s)\right| \leq a_{i j}^{M} \mu_{i j}^{M}\|\varphi\|+\sum_{C_{k l} \in N_{r}(i, j)} \overline{C_{i j}^{k l}} \\
& \times \int_{0}^{+\infty}\left|K_{i j}(u)\right|\left|f\left[\varphi_{k l}(s-u)\right]\right| \Delta u\left|\varphi_{i j}(s)\right| \\
& +\sum_{C_{k l} \in N_{s}(i, j)} \overline{D_{i j}^{k l}} \\
& \times \int_{0}^{+\infty}\left|J_{i j}(u)\right|\left|g\left[\varphi_{k l}^{\Delta}(s-u)\right]\right| \Delta u\left|\varphi_{i j}(s)\right| \\
& \leq a_{i j}^{M} \mu_{i j}^{M}\|\varphi\|+\sum_{C_{k l} \in N_{r}(i, j)} \overline{C_{i j}^{k l}} \\
& \times \int_{0}^{+\infty}\left|K_{i j}(u)\right| \Delta u(L\|\varphi\|+|f(0)|)\|\varphi\| \\
& +\sum_{C_{k l} \in N_{s}(i, j)} \overline{D_{i j}^{k l}} \int_{0}^{+\infty}\left|J_{i j}(u)\right| \Delta u(l\|\varphi\|+|g(0)|)\|\varphi\| \\
& \leq\left(a_{i j}^{M} \mu_{i j}^{M}+\sum_{C_{k l} \in N_{r}(i, j)} \overline{C_{i j}^{k l}} K_{i j}^{0}(L\|\varphi\|+|f(0)|)\right. \\
& \left.+\sum_{C_{k l} \in N_{s}(i, j)} \overline{D_{i j}^{k l}} J_{i j}^{0}(l\|\varphi\|+|g(0)|)\right)\|\varphi\| \\
& \leq\left(a_{i j}^{M} \mu_{i j}^{M}+\sum_{C_{k l} \in N_{r}(i, j)} \overline{C_{i j}^{k l}} K_{i j}^{0}(2 A L+|f(0)|)\right. \\
& \left.+\sum_{C_{k l} \in N_{s}(i, j)} \overline{D_{i j}^{k l}} J_{i j}^{0}(2 A l+|g(0)|)\right) 2 A \\
& \leq 2 A \theta_{i j}^{0}, \quad i=1,2, \ldots, n, \quad j=1,2, \ldots, m .
\end{aligned}
$$


So

$$
\begin{aligned}
\left|\left(\Phi\left(\varphi-\varphi^{0}\right)\right)_{i j}(t)\right| & =\left|\int_{-\infty}^{t} e_{-a_{i j}}(t, \sigma(s)) F_{i j}^{\varphi}(s) \Delta s\right| \\
& \leq \int_{-\infty}^{t} e_{-a_{i j}}(t, \sigma(s))\left|F_{i j}^{\varphi}(s)\right| \Delta s \\
& \leq \int_{-\infty}^{t} e_{-a_{i j}^{m}}(t, \sigma(s)) 2 A \theta_{i j}^{0} \Delta s \\
& \leq \frac{2 A \theta_{i j}^{0}}{a_{i j}^{m}}, \\
i= & 1,2, \ldots, n, \quad j=1,2, \ldots, m .
\end{aligned}
$$

On the other hand, we have

$$
\begin{aligned}
& \left|\left(\Phi\left(\varphi-\varphi^{0}\right)^{\Delta}\right)_{i j}(t)\right| \\
& =\left|\left(\int_{-\infty}^{t} e_{-a_{i j}}(t, \sigma(s)) F_{i j}^{\varphi}(s) \Delta s\right)_{t}\right|^{\Delta} \\
& =\left|F_{i j}^{\varphi}(t)-a_{i j}(t) \int_{-\infty}^{t} e_{-a_{i j}}(t, \sigma(s)) F_{i j}^{\varphi}(s) \Delta s\right| \\
& \leq\left|F_{i j}^{\varphi}(t)\right|+\left|a_{i j}(t)\right| \int_{-\infty}^{t} e_{-a_{i j}}(t, \sigma(s))\left|F_{i j}^{\varphi}(s)\right| \Delta s \\
& \leq \frac{2 A \theta_{i j}^{0}}{a_{i j}^{m}+a_{i j}^{M} \frac{2 A \theta_{i j}^{0}}{a_{i j}^{m}}} \\
& =\left(1+\frac{a_{i j}^{M}}{a_{i j}^{m}}\right) 2 A \theta_{i j}^{0}, \quad i=1,2, \ldots, n, j=1,2, \ldots, m .
\end{aligned}
$$

In view of $\left(H_{5}\right)$, we have

$$
\left\|\Phi\left(\varphi-\varphi^{0}\right)\right\| \leq \max _{\substack{1 \leq i \leq n \\ 1 \leq j \leq m}}\left\{\frac{2 A \theta_{i j}^{0}}{a_{i j}^{m}},\left(1+\frac{a_{i j}^{M}}{a_{i j}^{m}}\right) 2 A \theta_{i j}^{0}\right\} \leq A ;
$$

that is, $\Phi(\varphi) \in \mathbb{X}_{0}$. Next, we show that $\Phi$ is a contraction. For $\varphi, \psi \in \mathbb{X}_{0}, i=1,2, \ldots, n, j=1,2, \ldots, m$, denote

$$
\begin{aligned}
G_{i j}^{1}(s, \varphi, \psi)= & -\sum_{C_{k l} \in N_{r}(i, j)} C_{i j}^{k l}(s) \\
& \times\left[\int_{0}^{+\infty} K_{i j}(u) f\left[\varphi_{k l}(s-u)\right] \Delta u \varphi_{i j}(s)\right. \\
& \left.\quad-\int_{0}^{+\infty} K_{i j}(u) f\left[\psi_{k l}(s-u)\right] \Delta u \psi_{i j}(s)\right], \\
& i=1,2, \ldots, n, \quad j=1,2, \ldots, m,
\end{aligned}
$$

$$
\begin{aligned}
& G_{i j}^{2}(s, \varphi, \psi) \\
& =a_{i j}(s) \int_{s-\mu_{i j}(s)}^{s}\left[\varphi_{i j}^{\Delta}(u)-\psi_{i j}^{\Delta}(u)\right] \Delta u \\
& \quad-\sum_{C_{k l} \in N_{s}(i, j)} D_{i j}^{k l}(s) \\
& \times\left[\int_{0}^{+\infty} J_{i j}(u) g\left[\varphi_{k l}^{\Delta}(s-u)\right] \Delta u \varphi_{i j}(s)\right. \\
& \left.-\int_{0}^{+\infty} J_{i j}(u) g\left[\psi_{k l}^{\Delta}(s-u)\right] \Delta u \psi_{i j}(s)\right], \\
& i=1,2, \ldots, n, \quad j=1,2, \ldots, m .
\end{aligned}
$$

So

$$
\begin{aligned}
& \left|G_{i j}^{1}(s, \varphi, \psi)\right| \\
& =\mid-\sum_{C_{k l} \in N_{r}(i, j)} C_{i j}^{k l}(s) \\
& \quad \times \mid \int_{0}^{+\infty} K_{i j}(u) f\left[\varphi_{k l}(s-u)\right] \Delta u \varphi_{i j}(s) \\
& \left.\quad-\int_{0}^{+\infty} K_{i j}(u) f\left[\psi_{k l}(s-u)\right] \Delta u \psi_{i j}(s)\right] \mid \\
& \quad \sum_{C_{k l} \in N_{r}(i, j)}\left|C_{i j}^{k l}(s)\right| \\
& \quad \times \mid \int_{0}^{+\infty} K_{i j}(u)\left[f\left(\varphi_{k l}(s-u)\right)-f\left(\psi_{k l}(s-u)\right)\right] \\
& \quad \times \quad \int_{i j}^{+\infty}\left|K_{i j}(u)\right| f\left(\varphi_{k l}(s-u)\right) \\
& \quad \sum_{0}^{+\infty}\left|K_{i j}(u)\right|\left(\left|f\left(\psi_{k l}(s-u)\right)-f(0)\right|+|f(0)|\right) \\
& \quad-\int_{i j}^{+\infty} K_{i j}(u) f\left(\psi_{k l}(s-u)\right) \Delta u\left(\psi_{i j}(s)-\varphi_{i j}(s)\right) \mid
\end{aligned}
$$




$$
\begin{aligned}
& \times\left(L \int_{0}^{+\infty}\left|K_{i j}(u)\right| \Delta u\|\varphi-\psi\|\|\varphi\|\right. \\
& \left.+\int_{0}^{+\infty}\left|K_{i j}(u)\right| \Delta u(L\|\psi\|+|f(0)|)\|\varphi-\psi\|\right) \\
& \leq \sum_{C_{k l} \in N_{r}(i, j)} \overline{C_{i j}^{k l}}\left(K_{i j}^{0}(4 A L+|f(0)|)\right)\|\psi-\varphi\|, \\
& i=1,2, \ldots, n, \quad j=1,2, \ldots, m .
\end{aligned}
$$

Similarly,

$$
\begin{aligned}
& \left|G_{i j}^{2}(s, \varphi, \psi)\right| \\
& \quad \leq\left(a_{i j}^{M} \mu_{i j}^{M}+\sum_{C_{k l} \in N_{s}(i, j)} \overline{D_{i j}^{k l}}\left(J_{i j}^{0}(4 A l+|g(0)|)\right)\right)\|\psi-\varphi\|,
\end{aligned}
$$

so

$$
\begin{aligned}
& \left|G_{i j}^{1}(s, \varphi, \psi)+G_{i j}^{2}(s, \varphi, \psi)\right| \\
& \quad \leq\left|G_{i j}^{1}(s, \varphi, \psi)\right|+\left|G_{i j}^{2}(s, \varphi, \psi)\right| \\
& \quad \leq \mid a_{i j}^{M} \mu_{i j}^{M}+\sum_{C_{k l} \in N_{r}(i, j)} \overline{C_{i j}^{k l}} K_{i j}^{0}(4 A L+|f(0)|) \\
& \quad=\theta_{i j}\|\psi-\varphi\|, \quad i=1,2, \ldots, n, \quad j=1,2, \ldots, m, \\
& \left.\quad+\sum_{D_{i j}^{k l}} J_{i j}^{0}(4 A l+|g(0)|)\right)\|\psi-\varphi\| \\
& \quad=\left|\int_{-\infty}^{t} e_{-a_{i j}}(t, \sigma(s))\left(G_{i j}^{1}(s, \varphi, \psi)+G_{i j}^{2}(s, \varphi, \psi)\right) \Delta s\right| \\
& \quad \leq \int_{-\infty}^{t} e_{-a_{i j}}(t, \sigma(s))\left|G_{i j}^{1}(s, \varphi, \psi)+G_{i j}^{2}(s, \varphi, \psi)\right| \Delta s \\
& \quad \theta_{i j} e_{-\infty}\|\psi-\varphi\|, \quad i=1,2, \ldots, n, \quad j=1,2, \ldots, m, \\
& |(\Phi)| \Phi \psi)_{i j}^{\Delta}(t) \mid
\end{aligned}
$$

By $\left(H_{5}\right)$, we have

$$
\max _{\substack{1 \leq i \leq n \\ 1 \leq j \leq m}}\left\{\frac{1}{a_{i j}^{m}} \theta_{i j},\left(1+\frac{a_{i j}^{M}}{a_{i j}^{m}}\right) \theta_{i j}\right\}\|\psi-\varphi\|<\|\psi-\varphi\|,
$$

which implies that

$$
\|\Phi \varphi-\Phi \psi\|<\|\varphi-\psi\|
$$

It follows that $\Phi$ is a contraction. Therefore, $\Phi$ has a fixed point in $\mathbb{X}_{0}$; that is, (3) has a unique periodic solution in $\mathbb{X}_{0}$. This completes the proof of Theorem 16.

\section{Exponential Stability of the Periodic Solution}

In this section, we will discuss the exponential stability of the periodic solution of (3).

Definition 17. The periodic solution $x(t)=\left\{x_{i j}(t)\right\}$ of system (3) with initial value $\psi(t)=\left\{\psi_{i j}(t)\right\}$ is said to be globally exponentially stable, if there exists a positive constant $\lambda$ with $\ominus \lambda \in \mathscr{R}^{+}$and $M>1$ such that every solution $y(t)=\left\{y_{i j}(t)\right\}$ of (3) with any initial value $\varphi(t)=\left\{\varphi_{i j}(t)\right\}$ satisfies

$$
\|x(t)-y(t)\|_{1} \leq M e_{\ominus \lambda}\left(t, t_{0}\right)\|\phi\|, \quad \forall t \in(0,+\infty)_{\mathbb{T}},
$$


where

$$
\begin{aligned}
& \|x(t)-y(t)\|_{1}=\max \left\{\max _{i j}\left\{\left|x_{i j}(t)-y_{i j}(t)\right|\right\},\right. \\
& \left.\max _{i j}\left\{\left|x_{i j}^{\Delta}(t)-y_{i j}^{\Delta}(t)\right|\right\}\right\}, \\
& \|\phi\|=\max \left\{\sup _{s \in(-\infty, 0]_{\mathbb{T}}} \max _{i j}\left\{\left|\psi_{i j}(s)-\varphi_{i j}(s)\right|\right\},\right. \\
& \left.\sup _{s \in(-\infty, 0]_{\mathbb{T}}} \max _{i j}\left\{\left|\psi_{i j}^{\Delta}(s)-\varphi_{i j}^{\Delta}(s)\right|\right\}\right\}, \\
& i=1,2, \ldots, n, \quad j=1,2, \ldots, m, \\
& t_{0}=\max _{i=1}\left\{(-\infty, 0]_{\mathbb{T}}\right\} .
\end{aligned}
$$

Theorem 18. Suppose that all of the conditions in Theorem 16 are satisfied; then the unique periodic solution of (3) is globally exponentially stable.

Proof. By Theorem 16, (3) has an $\omega$-periodic solution $x(t)=$ $\left\{x_{i j}(t)\right\}$ with initial condition $\psi(t)=\left\{\psi_{i j}(t)\right\}, i=1,2, \ldots, n$, $j=1,2, \ldots, m$. Suppose that $y(t)=\left\{y_{i j}(t)\right\}$ is an arbitrary solution of (3), associated with the initial value $\varphi(t)=\left\{\varphi_{i j}(t)\right\}$, $i=1,2, \ldots, n, j=1,2, \ldots, m$. Let $z(t)=y(t)-x(t)$ and $\phi(t)=\varphi(t)-\psi(t)$. Then it follows from system (3) that

$$
\begin{aligned}
z_{i j}^{\Delta}(t) & +a_{i j}(t) z_{i j}(t) \\
= & a_{i j}(t) \int_{t-\mu_{i j}(t)}^{t} z_{i j}^{\Delta}(s) \Delta s \\
& -\sum_{C_{k l} \in N_{r}(i, j)} C_{i j}^{k l}(t) \\
\times & {\left[\int_{0}^{+\infty} K_{i j}(u) f\left[x_{k l}(t-u)\right] \Delta u x_{i j}(t)\right.} \\
& \left.-\int_{0}^{+\infty} K_{i j}(u) f\left[y_{k l}(t-u)\right] \Delta u y_{i j}(t)\right] \\
& \quad \sum_{k l} \in N_{s}(i, j) \\
\times & {\left[\int_{i j}^{k l}(t)\right.} \\
& -\int_{0}^{+\infty} J_{i j}(u) g\left[x_{k l}^{\Delta}(t-u)\right] \Delta u x_{i j}(t)
\end{aligned}
$$

where $z_{i j}(t)=y_{i j}(t)-x_{i j}(t), i=1,2, \ldots, n, j=1,2, \ldots, m$ and the initial condition of (46) is

$$
\phi_{i j}(s)=\varphi_{i j}(s)-\psi_{i j}(s),
$$

$s \in(-\infty, 0]_{\mathbb{T}}, \quad i=1,2, \ldots, n, \quad j=1,2, \ldots, m$.
Let $S_{i j}$ and $T_{i j}$ be defined as follows:

$$
\begin{aligned}
S_{i j}(\beta) & =a_{i j}^{m}-\beta-\exp \left(\beta \sup _{s \in \mathbb{T}}(\mu(s))\right) A_{i j}(\beta), \\
T_{i j}(\beta)= & a_{i j}^{m}-\beta \\
& -\left(a_{i j}^{M} \exp \left(\beta \sup _{s \in \mathbb{T}}(\mu(s))\right)+a_{i j}^{m}-\beta\right) A_{i j}(\beta),
\end{aligned}
$$

where

$$
\begin{gathered}
A_{i j}(\beta) \\
=a_{i j}^{M} \mu_{i j}^{M}+\sum_{C_{k l} \in N_{r}(i, j)} \overline{C_{i j}^{k l}}\left[(A L+|f(0)|) \int_{0}^{+\infty}\left|K_{i j}(u)\right| \Delta u\right. \\
\left.+A L \int_{0}^{+\infty}\left|K_{i j}(u)\right| \exp (\beta u) \Delta u\right] \\
+\sum_{C_{k l} \in N_{s}(i, j)} \overline{D_{i j}^{k l}}\left[(A l+|g(0)|) \int_{0}^{+\infty}\left|J_{i j}(u)\right| \Delta u\right. \\
\left.+A l \int_{0}^{+\infty}\left|J_{i j}(u)\right| \exp (\beta u) \Delta u\right], \\
i=1,2, \ldots, n, \quad j=1,2, \ldots, m .
\end{gathered}
$$

By $\left(H_{5}\right)$, for $i=1,2, \ldots, n, j=1,2, \ldots, m$, we have

$$
\begin{array}{r}
S_{i j}(0)=a_{i j}^{m}-A_{i j}(0) \geq a_{i j}^{m}-\theta_{i j}^{0}>0, \\
T_{i j}(0)=a_{i j}^{m}-\left(a_{i j}^{M}+a_{i j}^{m}\right) A_{i j}(0) \\
\geq a_{i j}^{m}-\left(a_{i j}^{M}+a_{i j}^{m}\right) \theta_{i j}^{0}>0 .
\end{array}
$$

Since $S_{i j}$ and $T_{i j}$ are continuous on $[0,+\infty)$ and $S_{i j}(\beta), T_{i j}(\beta) \rightarrow+\infty$ as $\beta \rightarrow+\infty$, there exist $\lambda_{i j}>0$ and $s_{i j}>0$ such that $S_{i j}\left(\lambda_{i j}\right)=0$ and $S_{i j}(\beta)>0$ for all $\beta \in\left(0, \lambda_{i j}\right), T_{i j}\left(\varsigma_{i j}\right)=0$, and $T_{i j}(\beta)>0$ for all $\beta \in\left(0, \varsigma_{i j}\right)$. Take $\lambda^{*}=\min _{1 \leq i \leq n, 1 \leq j \leq m}\left\{\lambda_{i j}\right.$, $\left.\varsigma_{i j}\right\}$; then

$$
\begin{array}{r}
S_{i j}\left(\lambda^{*}\right) \geq 0, \quad T_{i j}\left(\lambda^{*}\right) \geq 0, \\
i=1,2, \ldots, n, \quad j=1,2, \ldots, m .
\end{array}
$$

So, we can choose a positive constant $0<\lambda<\min \left\{\lambda^{*}, a_{i j}^{m}, i=\right.$ $1,2, \ldots, n, j=1,2, \ldots, m\}$ such that

$$
\begin{gathered}
S_{i j}(\lambda)>0, \quad T_{i j}(\lambda)>0, \\
i=1,2, \ldots, n, \quad j=1,2, \ldots, m,
\end{gathered}
$$


which implies that

$$
\begin{gathered}
\frac{\exp \left(\lambda \sup _{s \in \mathbb{T}}(\mu(s))\right) A_{i j}(\lambda)}{a_{i j}^{m}-\lambda}<1, \\
i=1,2, \ldots, n, \quad j=1,2, \ldots, m, \\
\left(1+\frac{a_{i j}^{M} \exp \left(\lambda \sup _{s \in \mathbb{T}}(\mu(s))\right)}{a_{i j}^{m}-\lambda}\right) A_{i j}(\lambda)<1, \\
i=1,2, \ldots, n, \quad j=1,2, \ldots, m .
\end{gathered}
$$

Let $M=\max _{1 \leq i \leq n, 1 \leq j \leq m}\left\{a_{i j}^{m} / A_{i j}(0)\right\}$; by $\left(H_{5}\right)$, we have $M>1$. It is obvious that

$$
\|Z(t)\|_{1}=\|\phi(t)\|_{1} \leq M\|\phi\| e_{\ominus \lambda}\left(t, t_{0}\right), \quad \forall t \in(-\infty, 0]_{\mathbb{T}},
$$

where $\lambda \in \mathscr{R}^{+}$. In the following, we will show that

$$
\|Z(t)\|_{1}=\|\phi(t)\|_{1} \leq M\|\phi\| e_{\ominus \lambda}\left(t, t_{0}\right), \quad \forall t \in(0,+\infty)_{\mathbb{T}} .
$$

holds for all $t \in(0,+\infty)_{\mathbb{T}}$.

For this aim, we show that for any $P>1$,

$$
\|Z(t)\|_{1}<P M\|\phi\| e_{\ominus \lambda}\left(t, t_{0}\right), \quad \forall t \in(0,+\infty)_{\mathbb{J}} .
$$

If (57) is not true, then there must be $t_{1} \in(0,+\infty)_{\mathbb{T}}$ such that

$$
\begin{aligned}
\left\|Z\left(t_{1}\right)\right\| & =\max \left\{\left\|Z\left(t_{1}\right)\right\|,\left\|Z^{\Delta}\left(t_{1}\right)\right\|\right\} \\
& =\max _{\substack{1 \leq j \leq n \\
1 \leq j \leq m}}\left\{\left|Z_{i j}\left(t_{1}\right)\right|,\left|Z_{i j}^{\Delta}\left(t_{1}\right)\right|\right\} \\
& \geq P M\|\phi\| e_{\ominus \lambda}\left(t_{1}, t_{0}\right), \\
& \|Z(t)\| \leq P M\|\phi\| e_{\ominus \lambda}\left(t, t_{0}\right), \\
& t \in\left(-\infty, t_{1}\right]_{\mathbb{T}}, \quad t_{0} \in(-\infty, 0)_{\mathbb{V}} .
\end{aligned}
$$

Therefore, there must exist a constant $c \geq 1$ such that

$$
\begin{aligned}
\left\|Z\left(t_{1}\right)\right\|= & \max \left\{\left\|Z\left(t_{1}\right)\right\|,\left\|Z^{\Delta}\left(t_{1}\right)\right\|\right\} \\
= & \max \left\{\left|Z_{i j}\left(t_{1}\right)\right|,\left|Z_{i j}^{\Delta}\left(t_{1}\right)\right|\right\} \\
= & c P M\|\phi\| e_{\ominus \lambda}\left(t_{1}, t_{0}\right), \\
& \|Z(t)\| \leq c P M\|\phi\| e_{\ominus \lambda}\left(t, t_{0}\right), \\
t & \in\left(-\infty, t_{1}\right]_{\mathbb{T}}, \quad t_{0} \in(-\infty, 0)_{\mathbb{T}} .
\end{aligned}
$$

Multiplying both sides of (46) by $e_{-a_{i j}}\left(t_{0}, \sigma(s)\right)$ and integrating over $\left[t_{0}, t\right]$, by Lemma 15 , for $i=1,2, \ldots, n, j=$ $1,2, \ldots, m$, we get

$$
\begin{aligned}
& z_{i j}(t)=\phi_{i j}\left(t_{0}\right) e_{-a_{i j}}\left(t, t_{0}\right) \\
& +\int_{t_{0}}^{t} e_{-a_{i j}}(t, \sigma(s)) \\
& \times\left\{a_{i j}(s) \int_{s-\mu_{i j}(s)}^{s} x_{i j}^{\Delta}(u) \Delta u-\sum_{C_{k l} \in N_{r}(i, j)} C_{i j}^{k l}(s)\right. \\
& \times\left[\int_{0}^{+\infty} K_{i j}(u) f\left[x_{k l}(s-u)\right] \Delta u x_{i j}(s)\right. \\
& \left.-\int_{0}^{+\infty} K_{i j}(u) f\left[y_{k l}(s-u)\right] \Delta u y_{i j}(s)\right] \\
& -\sum_{C_{k l} \in N_{s}(i, j)} D_{i j}^{k l}(s) \\
& \times\left[\int_{0}^{+\infty} J_{i j}(u) g\left[x_{k l}^{\Delta}(s-u)\right] \Delta u x_{i j}(s)\right. \\
& \left.\left.-\int_{0}^{+\infty} J_{i j}(u) g\left[y_{k l}^{\Delta}(s-u)\right] \Delta u y_{i j}(s)\right]\right\} \Delta s .
\end{aligned}
$$

It follows from (60), (61), and the assumptions of the theorem that

$$
\begin{aligned}
& \left|z_{i j}\left(t_{1}\right)\right| \\
& \leq\|\phi\| e_{-a_{i j}}\left(t_{1}, t_{0}\right)+\int_{t_{0}}^{t_{1}} e_{-a_{i j}}\left(t_{1}, \sigma(s)\right) \\
& \times\left\{a_{i j}^{M} \int_{s-\mu_{i j}(s)}^{s}\left|z_{i j}^{\Delta}(u)\right| \Delta u+\sum_{C_{k l} \in N_{r}(i, j)} \overline{C_{i j}^{k l}}\right. \\
& \quad \times \mid \int_{0}^{+\infty} K_{i j}(u) f\left[x_{k l}(s-u)\right] \Delta u x_{i j}(s) \\
& \quad-\int_{0}^{+\infty} K_{i j}(u) f\left[y_{k l}(s-u)\right] \Delta u y_{i j}(s) \mid \\
& +\quad \sum_{k l} \overline{D_{i j}^{k l}(i, j)} \\
& \quad \times \mid \int_{0}^{+\infty} J_{i j}(u) g\left[x_{k l}^{\Delta}(s-u)\right] \Delta u x_{i j}(s) \\
& \left.\quad-\int_{0}^{+\infty} J_{i j}(u) g\left[y_{k l}^{\Delta}(s-u)\right] \Delta u y_{i j}(s) \mid\right\} \Delta s
\end{aligned}
$$




$$
\begin{aligned}
& \leq\|\phi\| e_{-a_{i j}}\left(t_{1}, t_{0}\right)+\int_{t_{0}}^{t_{1}} e_{-a_{i j}}\left(t_{1}, \sigma(s)\right) \\
& \times\left\{a_{i j}^{M} \int_{s-\mu_{i j}(s)}^{s}\left|z_{i j}^{\Delta}(u)\right| \Delta u\right. \\
& +\sum_{C_{k l} \in N_{r}(i, j)} \overline{C_{i j}^{k l}} \\
& \times\left[A L \int_{0}^{+\infty}\left|K_{i j}(u)\right|\left|x_{k l}(s-u)-y_{k l}(s-u)\right| \Delta u\right. \\
& +(A L+|f(0)|) \\
& \left.\times \int_{0}^{+\infty}\left|K_{i j}(u)\right| \Delta u\left|x_{k l}(s)-y_{k l}(s)\right|\right] \\
& +\sum_{C_{k l} \in N_{s}(i, j)} \overline{D_{i j}^{k l}} \\
& \times\left[A l \int_{0}^{+\infty}\left|J_{i j}(u)\right|\left|x_{k l}^{\Delta}(s-u)-y_{k l}^{\Delta}(s-u)\right| \Delta u\right. \\
& +(A l+|g(0)|) \\
& \left.\left.\times \int_{0}^{+\infty}\left|J_{i j}(u)\right| \Delta u\left|x_{k l}(s)-y_{k l}(s)\right|\right]\right\} \Delta s \\
& \leq\|\phi\| e_{-a_{i j}}\left(t_{1}, t_{0}\right)+c P M\|\phi\| e_{\ominus \lambda}\left(t_{1}, t_{0}\right) a_{i j}^{M} \mu_{i j}^{M} \\
& +\int_{t_{0}}^{t_{1}} c P M\|\phi\| e_{-a_{i j}}\left(t_{1}, \sigma(s)\right) e_{\ominus \lambda}\left(t_{1}, t_{0}\right) e_{\lambda}\left(t_{1}, \sigma(s)\right) \\
& \times\left\{\sum_{C_{k l} \in N_{r}(i, j)} \overline{C_{i j}^{k l}}\right. \\
& \times\left[A L \int_{0}^{+\infty}\left|K_{i j}(u)\right| e_{\lambda}(\sigma(s), s-u)\right. \\
& \left.+(A L+|f(0)|) \int_{0}^{+\infty}\left|K_{i j}(u)\right| e_{\lambda}(\sigma(s), s) \Delta u\right] \\
& +\sum_{C_{k l} \in N_{s}(i, j)} \overline{D_{i j}^{k l}} \\
& \times\left[A l \int_{0}^{+\infty}\left|J_{i j}(u)\right| e_{\lambda}(\sigma(s), s-u)\right. \\
& +(A l+|g(0)|) \\
& \left.\left.\times \int_{0}^{+\infty}\left|J_{i j}(u)\right| e_{\lambda}(\sigma(s), s) \Delta u\right]\right\} \Delta s
\end{aligned}
$$$$
+\int_{t_{0}}^{t_{1}} e_{-a_{i j}}\left(t_{1}, \sigma(s)\right) e_{\lambda}\left(t_{1}, t_{0}\right)
$$$$
\times\left\{\sum_{C_{k l} \in N_{r}(i, j)} \overline{C_{i j}^{k l}}\right.
$$$$
\times\left[A L \int_{0}^{+\infty}\left|K_{i j}(u)\right|\right.
$$$$
\times \exp \left(\lambda\left(u+\sup _{s \in \mathbb{T}} \mu(s)\right)\right) \Delta u
$$$$
+(A L+|f(0)|)
$$$$
\left.\times \int_{0}^{+\infty}\left|K_{i j}(u)\right| \exp (\lambda \mu(s)) \Delta u\right]
$$$$
+\sum_{C_{k l} \in N_{s}(i, j)} \overline{D_{i j}^{k l}}
$$$$
\times\left[A l \int_{0}^{+\infty}\left|J_{i j}(u)\right| \exp \left(\lambda\left(u+\sup _{s \in \mathbb{T}} \mu(s)\right)\right) \Delta u\right.
$$$$
+(A l+|g(0)|)
$$$$
\left.\left.\left.\times \int_{0}^{+\infty}\left|J_{i j}(u)\right| \exp (\lambda \mu(s)) \Delta u\right]\right\}\right\}
$$

$\leq c P M\|\phi\| e_{\ominus \lambda}\left(t_{1}, t_{0}\right)$

$$
\begin{gathered}
\times\left\{\frac{1}{M} e_{-a_{i j} \oplus \lambda}\left(t_{1}, t_{0}\right)+a_{i j}^{M} \mu_{i j}^{M}+\exp \left(\lambda \sup _{s \in \mathbb{T}} \mu(s)\right)\right. \\
\times\left\{\sum_{C_{k l} \in N_{r}(i, j)} \overline{C_{i j}^{k l}}\right. \\
\times\left[A L \int_{0}^{+\infty}\left|K_{i j}(u)\right| \exp (\lambda u) \Delta u\right. \\
\left.\quad \times \int_{0}^{+\infty}\left|K_{i j}(u)\right| \Delta u\right] \\
+\sum_{C_{k l} \in N_{s}(i, j)} \overline{D_{i j}^{k l}}(A L+|f(0)|) \\
\times\left[\int_{0}^{+\infty}\left|J_{i j}(u)\right| \exp (\lambda u) \Delta u+(A l+|g(0)|)\right. \\
\left.\left.\times \int_{0}^{+\infty}\left|J_{i j}(u)\right| \Delta u\right]\right\} \int_{t_{0}}^{t_{1}} e_{-a_{i j}}\left(t_{1}, \sigma(s)\right) \Delta s
\end{gathered}
$$$$
\times\left\{\frac{1}{c P M} e_{-a_{i j}}\left(t_{1}, t_{0}\right) e_{\ominus \lambda}\left(t_{0}, t_{1}\right)+a_{i j}^{M} \mu_{i j}^{M}\right.
$$ 


$$
\begin{aligned}
\leq c P M & \|\phi\| e_{\ominus \lambda}\left(t_{1}, t_{0}\right) \\
\times\{( & \left.\frac{1}{M}-\frac{\exp \left(\lambda \sup _{s \in \mathbb{T}}(\mu(s))\right) A_{i j}(\lambda)}{a_{i j}^{m}-\lambda}\right) \\
\quad \times e_{-a_{i j} \oplus \lambda}\left(t_{1}, t_{0}\right) & \\
+ & \left.\frac{\exp \left(\lambda \sup _{s \in \mathbb{T}}(\mu(s))\right) A_{i j}(\lambda)}{a_{i j}^{m}-\lambda}\right\}
\end{aligned}
$$

$<c P M\|\phi\| e_{\ominus \lambda}\left(t_{1}, t_{0}\right)$.

A direct differentiation of (61) gives

$$
\begin{aligned}
& z_{i j}^{\Delta}(t)=-a_{i j}(t) z_{i j}(t)+a_{i j}(t) \int_{t-\mu_{i j}(t)}^{t} z_{i j}^{\Delta}(s) \Delta s \\
&-\sum_{C_{k l} \in N_{r}(i, j)} C_{i j}^{k l}(t) \\
& \times\left[\int_{0}^{+\infty} K_{i j}(u) f\left[x_{k l}(t-u)\right] \Delta u x_{i j}(t)\right. \\
&\left.\quad-\int_{0}^{+\infty} K_{i j}(u) f\left[y_{k l}(t-u)\right] \Delta u y_{i j}(t)\right] \\
&-\sum_{C_{k l} \in N_{s}(i, j)} D_{i j}^{k l}(t) \\
& \times\left[\int_{0}^{+\infty} J_{i j}(u) g\left[x_{k l}^{\Delta}(t-u)\right] \Delta u x_{i j}(t)\right. \\
&\left.\quad-\int_{0}^{+\infty} J_{i j}(u) g\left[y_{k l}^{\Delta}(t-u)\right] \Delta u y_{i j}(t)\right], \\
& i=1,2, \ldots, n, \quad j=1,2, \ldots, m .
\end{aligned}
$$

Thus, we have by (53), (54), (60), and (63)

$$
\begin{aligned}
& \left|z_{i j}^{\Delta}\left(t_{1}\right)\right| \\
& \leq a_{i j}^{M}\left|z_{i j}\left(t_{1}\right)\right|+a_{i j}^{M} \int_{t_{1}-\mu_{i j}\left(t_{1}\right)}^{t_{1}}\left|z_{i j}^{\Delta}(s)\right| \Delta s \\
& +\sum_{C_{k l} \in N_{r}(i, j)} \overline{C_{i j}^{k l}} \\
& \quad \times\left[A L \int_{0}^{+\infty}\left|K_{i j}(u)\right|\left|x_{k l}\left(t_{1}-u\right)-y_{k l}\left(t_{1}-u\right)\right| \Delta u\right. \\
& \quad+(A L+|f(0)|) \\
& \left.\quad \times \int_{0}^{+\infty}\left|K_{i j}(u)\right| \Delta u\left|x_{k l}\left(t_{1}\right)-y_{k l}\left(t_{1}\right)\right|\right] \\
& +\sum_{C_{k l} \in N_{s}(i, j)} \frac{D_{i j}^{k l}}{}
\end{aligned}
$$

$$
\begin{aligned}
& \times\left[A l \int_{0}^{+\infty}\left|J_{i j}(u)\right|\left|x_{k l}^{\Delta}\left(t_{1}-u\right)-y_{k l}^{\Delta}\left(t_{1}-u\right)\right| \Delta u\right. \\
& \left.+(A l+|g(0)|) \int_{0}^{+\infty}\left|J_{i j}(u)\right| \Delta u\left|x_{k l}\left(t_{1}\right)-y_{k l}\left(t_{1}\right)\right|\right] \\
& \leq a_{i j}^{M}\left|z_{i j}\left(t_{1}\right)\right|+P M\|\phi\| e_{\ominus \lambda}\left(t_{1}, t_{0}\right) \\
& \times\left\{a_{i j}^{M} \mu_{i j}^{M}+\sum_{C_{k l} \in N_{r}(i, j)} \overline{C_{i j}^{k l}}\right. \\
& \times\left[A L \times \int_{0}^{+\infty}\left|K_{i j}(u)\right| e_{\lambda}\left(t_{1}, t_{1}-u\right) \Delta u\right. \\
& \left.+(A L+|f(0)|) \int_{0}^{+\infty}\left|K_{i j}(u)\right| e_{\lambda}\left(t_{1}, t_{1}\right) \Delta u\right] \\
& +\sum_{C_{k l} \in N_{s}(i, j)} \overline{D_{i j}^{k l}} \\
& \times\left[A l \int_{0}^{+\infty}\left|J_{i j}(u)\right| e_{\lambda}\left(t_{1}, t_{1}-u\right) \Delta u\right. \\
& \left.\left.+(A l+|g(0)|) \int_{0}^{+\infty}\left|J_{i j}(u)\right| e_{\lambda}\left(t_{1}, t_{1}\right) \Delta u\right]\right\} \\
& \leq a_{i j}^{M}\left|z_{i j}\left(t_{1}\right)\right|+c P M\|\phi\| e_{\ominus \lambda}\left(t_{1}, t_{0}\right) \\
& \times\left\{a_{i j}^{M} \mu_{i j}^{M}+\sum_{C_{k l} \in N_{r}(i, j)} \overline{C_{i j}^{k l}}\right. \\
& \times\left[A L \int_{0}^{+\infty}\left|K_{i j}(u)\right| \exp (\lambda u) \Delta u\right. \\
& \left.+(A L+|f(0)|) \int_{0}^{+\infty}\left|K_{i j}(u)\right| \Delta u\right] \\
& +\sum_{C_{k l} \in N_{s}(i, j)} \overline{D_{i j}^{k l}} \\
& \times\left[A l \int_{0}^{+\infty}\left|J_{i j}(u)\right| \exp (\lambda u) \Delta u\right. \\
& \left.\left.+(A l+|g(0)|) \int_{0}^{+\infty}\left|J_{i j}(u)\right| \Delta u\right]\right\} \\
& \leq a_{i j}^{M}\left|z_{i j}\left(t_{1}\right)\right|+c P M\|\phi\| e_{\ominus \lambda}\left(t_{1}, t_{0}\right) A_{i j}(\lambda) \\
& \leq a_{i j}^{M} \mid c P M\|\phi\| e_{\ominus \lambda}\left(t_{1}, t_{0}\right) \\
& \times\left\{\frac{1}{M} e_{-a_{i j} \oplus \lambda}\left(t_{1}, t_{0}\right)+\exp \left(\lambda \sup _{s \in \mathbb{T}} \mu(s)\right) A_{i j}(\lambda)\right. \\
& \left.\times \int_{t_{0}}^{t_{1}} e_{-a_{i j} \oplus \lambda}\left(t_{1}, \sigma(s)\right) \Delta s\right\} \mid
\end{aligned}
$$




$$
\begin{aligned}
& +c P M\|\phi\| e_{\ominus \lambda}\left(t_{1}, t_{0}\right) A_{i j}(\lambda) \\
\leq & c P M\|\phi\| e_{\ominus \lambda}\left(t_{1}, t_{0}\right) \\
& \times\left\{\frac{a_{i j}^{M}}{M} e_{-a_{i j} \oplus \lambda}\left(t_{1}, t_{0}\right)+a_{i j}^{M} \exp \left(\lambda \sup _{s \in \mathbb{T}} \mu(s)\right) A_{i j}(\lambda)\right. \\
\leq & \left.\times \int_{t_{0}}^{t_{1}} e_{-a_{i j} \oplus \lambda}\left(t_{1}, \sigma(s)\right) \Delta s+A_{i j}(\lambda)\right\} \\
\times & \| \frac{a_{i j}^{M}}{M} e_{-a_{i j} \oplus \lambda}\left(t_{1}, t_{0}\right)+e_{\ominus \lambda}\left(t_{1}, t_{0}\right) \\
& \left.\times\left(\frac{e_{-a_{i j} \oplus \lambda}\left(t_{1}, t_{0}\right)-1}{a_{i j}^{m}-\lambda}\right)+A_{i j}(\lambda)\right\} \\
\leq & \left.\times\left(\frac{e_{-a_{i j} \oplus \lambda}\left(t_{1}, t_{0}\right)-1}{\lambda-a_{i j}^{m}}\right)+A_{i j}(\lambda)\right\} \\
\times & \left.\|\phi\| e_{\ominus \lambda}\left(t_{1}, t_{0}\right)\right) A_{i j}(\lambda) \\
M & e_{-a_{i j} \oplus \lambda}\left(t_{1}, t_{0}\right)-a_{i j}^{M} \exp \left(\lambda \sup _{s \in \mathbb{T}} \mu(s)\right) A_{i j}(\lambda) \\
& \times(\lambda)
\end{aligned}
$$$$
\leq c P M\|\phi\| e_{\ominus \lambda}\left(t_{1}, t_{0}\right)
$$$$
\times\left\{\frac{a_{i j}^{M}}{M} e_{-a_{i j} \oplus \lambda}\left(t_{1}, t_{0}\right)+a_{i j}^{M} \exp \left(\lambda \sup _{s \in \mathbb{T}} \mu(s)\right) A_{i j}(\lambda)\right.
$$$$
\left.\times\left(\frac{e_{-a_{i j} \oplus \lambda}\left(t_{1}, t_{0}\right)-1}{a_{i j}^{m}-\lambda}\right)+A_{i j}(\lambda)\right\}
$$$$
\leq c P M\|\phi\| e_{\ominus \lambda}\left(t_{1}, t_{0}\right)
$$$$
\times\left\{\left(\frac{1}{M}-\frac{\exp \left(\lambda \sup _{s \in \mathbb{T}}(\mu(s))\right) A_{i j}(\lambda)}{a_{i j}^{m}-\lambda}\right)\right.
$$$$
\times a_{i j}^{M} e_{-a_{i j} \oplus \lambda}\left(t_{1}, t_{0}\right)
$$$$
\left.+\left(\frac{a_{i j}^{M} \exp \left(\lambda \sup _{s \in \mathbb{T}}(\mu(s))\right)}{a_{i j}^{m}-\lambda}+1\right) A_{i j}(\lambda)\right\}
$$

$<c P M\|\phi\| e_{\ominus \lambda}\left(t_{1}, t_{0}\right)$.

In view of (62) and (64), we obtain

$$
\|Z(t)\|_{1}<c P M\|\phi\| e_{\ominus \lambda}\left(t_{1}, t_{0}\right),
$$

which contradicts (59), and so (57) holds. Letting $P \rightarrow 1$, then (56) holds. Hence, the almost periodic solution of system
(3) is globally exponentially stable. The global exponential stability implies that the periodic solution is unique. The proof is complete.

\section{Some Examples}

Consider the following SICNNs model:

$$
\begin{aligned}
x_{i j}^{\Delta}(t)= & -a_{i j}(t) x_{i j}\left(t-\mu_{i j}(t)\right) \\
& -\sum_{C_{k l} \in N_{r}(i, j)} C_{i j}^{k l}(t) \\
& \times \int_{0}^{+\infty} K_{i j}(u) f\left[x_{k l}(t-u)\right] \Delta u x_{i j}(t) \\
& -\sum_{C_{k l} \in N_{s}(i, j)} D_{i j}^{k l}(t) \\
& \times \int_{0}^{+\infty} J_{i j}(u) g\left[x_{k l}^{\Delta}(t-u)\right] \Delta u x_{i j}(t)+I_{i j}(t), \\
&
\end{aligned}
$$

where $f(x)=2 \cos x, g(x)=2|x|, K_{i j}(u)=e^{-4 u}$, and $J_{i j}(u)=e^{-2 u}, i, j=1,2,3$.

Example 1. In system (66), let $\mathbb{T}=\mathbb{R}$; then $\mu(t) \equiv 0$, and take

$$
\begin{aligned}
& {\left[\begin{array}{lll}
a_{11}(t) & a_{12}(t) & a_{13}(t) \\
a_{21}(t) & a_{22}(t) & a_{23}(t) \\
a_{31}(t) & a_{32}(t) & a_{33}(t)
\end{array}\right]} \\
& \quad=\left[\begin{array}{lll}
4+|\sin t| & 5+|\sin 2 t| & 8+|\sin t| \\
6+|\cos t| & 6+|\sin t| & 7+|\cos t| \\
8+|\cos t| & 8+|\sin t| & 5+|\sin 2 t|
\end{array}\right],
\end{aligned}
$$$$
\left[\begin{array}{lll}
c_{11}(t) & c_{12}(t) & c_{13}(t) \\
c_{21}(t) & c_{22}(t) & c_{23}(t) \\
c_{31}(t) & c_{32}(t) & c_{33}(t)
\end{array}\right]
$$$$
=\left[\begin{array}{lll}
0.01|\sin 2 t| & 0.02|\sin 3 t| & 0.01|\sin 4 t| \\
0.01|\sin 2 t| & 0.01|\sin 3 t| & 0.01|\sin 4 t| \\
0.01|\sin 2 t| & 0.01|\sin 3 t| & 0.01|\sin 4 t|
\end{array}\right],
$$

$$
\left[\begin{array}{lll}
d_{11}(t) & d_{12}(t) & d_{13}(t) \\
d_{21}(t) & d_{22}(t) & d_{23}(t) \\
d_{31}(t) & d_{32}(t) & d_{33}(t)
\end{array}\right]
$$

$$
=\left[\begin{array}{lll}
0.01|\cos 2 t| & 0.01|\cos 3 t| & 0.02|\cos 4 t| \\
0.01|\cos 2 t| & 0.01|\cos 3 t| & 0.01|\cos 4 t| \\
0.01|\cos 2 t| & 0.01|\cos 3 t| & 0.01|\cos 4 t|
\end{array}\right],
$$

$$
\left[\begin{array}{lll}
I_{11}(t) & I_{12}(t) & I_{13}(t) \\
I_{21}(t) & I_{22}(t) & I_{23}(t) \\
I_{31}(t) & I_{32}(t) & I_{33}(t)
\end{array}\right]=\left[\begin{array}{lll}
\sin t & \sin t & \cos t \\
\sin t & \cos t & \cos t \\
\cos t & \cos t & \cos t
\end{array}\right],
$$




$$
\begin{aligned}
& {\left[\begin{array}{lll}
\mu_{11}(t) & \mu_{12}(t) & \mu_{13}(t) \\
\mu_{21}(t) & \mu_{22}(t) & \mu_{23}(t) \\
\mu_{31}(t) & \mu_{32}(t) & \mu_{33}(t)
\end{array}\right]} \\
& \quad=\left[\begin{array}{ccc}
0.02|\cos 2 t| & 0.01|\cos 3 t| & 0.01|\cos 4 t| \\
0.011|\cos 2 t| & 0.001|\cos 3 t| & 0.01|\cos 4 t| \\
0.01|\cos 2 t| & 0.01|\cos 3 t| & 0.02|\cos 4 t|
\end{array}\right] .
\end{aligned}
$$

Let $r=s=1, A=1 / 2$, and $\delta=1$. Obviously, $L=l=2$, $f(0)=2$ and $g(0)=0, K_{i j}^{0}=1 / 4, J_{i j}^{0}=1 / 2$,

$$
\begin{aligned}
& {\left[\begin{array}{rrr}
a_{11}^{M} & a_{12}^{M} & a_{13}^{M} \\
a_{21}^{M} & a_{22}^{M} & a_{23}^{M} \\
a_{31}^{M} & a_{32}^{M} & a_{33}^{M}
\end{array}\right]=\left[\begin{array}{lll}
5 & 6 & 9 \\
7 & 7 & 8 \\
9 & 9 & 6
\end{array}\right]} \\
& {\left[\begin{array}{rrr}
a_{11}^{m} & a_{12}^{m} & a_{13}^{m} \\
a_{21}^{m} & a_{22}^{m} & a_{23}^{m} \\
a_{31}^{m} & a_{32}^{m} & a_{33}^{m}
\end{array}\right]=\left[\begin{array}{lll}
4 & 5 & 8 \\
6 & 6 & 7 \\
8 & 8 & 5
\end{array}\right],} \\
& \begin{array}{c}
{\left[\begin{array}{lll}
\overline{c_{11}} & \overline{c_{12}} & \overline{c_{13}} \\
\overline{c_{21}} & \overline{c_{22}} & \overline{c_{23}} \\
\overline{c_{31}} & \frac{\bar{c}}{c_{32}} & \frac{\overline{c_{33}}}{}
\end{array}\right]=\left[\begin{array}{lll}
0.02 & 0.02 & 0.01 \\
0.01 & 0.01 & 0.01 \\
0.01 & 0.01 & 0.01
\end{array}\right],} \\
{\left[\begin{array}{lll}
\overline{d_{11}} & \overline{d_{12}} & \overline{d_{13}} \\
\frac{d_{21}}{d_{31}} & \frac{d_{22}}{d_{32}} & \frac{d_{23}}{d_{33}}
\end{array}\right]=\left[\begin{array}{lll}
0.01 & 0.01 & 0.02 \\
0.01 & 0.01 & 0.01 \\
0.01 & 0.01 & 0.01
\end{array}\right],}
\end{array} \\
& \begin{array}{c}
{\left[\begin{array}{lll}
\overline{c_{11}} & \overline{c_{12}} & \overline{c_{13}} \\
\overline{c_{21}} & \overline{c_{22}} & \overline{c_{23}} \\
\overline{c_{31}} & \frac{\bar{c}}{c_{32}} & \frac{\overline{c_{33}}}{}
\end{array}\right]=\left[\begin{array}{lll}
0.02 & 0.02 & 0.01 \\
0.01 & 0.01 & 0.01 \\
0.01 & 0.01 & 0.01
\end{array}\right],} \\
{\left[\begin{array}{lll}
\overline{d_{11}} & \overline{d_{12}} & \overline{d_{13}} \\
\frac{d_{21}}{d_{31}} & \frac{d_{22}}{d_{32}} & \frac{d_{23}}{d_{33}}
\end{array}\right]=\left[\begin{array}{lll}
0.01 & 0.01 & 0.02 \\
0.01 & 0.01 & 0.01 \\
0.01 & 0.01 & 0.01
\end{array}\right],}
\end{array} \\
& f(0)=2 \text { and } g(0)=0, K_{i j}^{0}=1 / 4, J_{i j}=1 / 2,
\end{aligned}
$$$$
\left[\begin{array}{lll}
\overline{I_{11}} & \overline{I_{12}} & \overline{I_{13}} \\
\overline{I_{21}} & \overline{I_{22}} & \overline{I_{23}} \\
I_{32} & \overline{I_{33}}
\end{array}\right]=\left[\begin{array}{lll}
1 & 1 & 1 \\
1 & 1 & 1 \\
1 & 1 & 1
\end{array}\right],
$$$$
\left[\begin{array}{lll}
\mu_{11}^{M} & \mu_{12}^{M} & \mu_{13}^{M} \\
\mu_{21}^{M} & \mu_{22}^{M} & \mu_{23}^{M} \\
\mu_{31}^{M} & \mu_{32}^{M} & \mu_{33}^{M}
\end{array}\right]=\left[\begin{array}{ccc}
0.02 & 0.01 & 0.01 \\
0.011 & 0.001 & 0.01 \\
0.01 & 0.01 & 0.02
\end{array}\right] \text {. }
$$

So

$$
\begin{aligned}
& {\left[\begin{array}{lll}
\theta_{11}^{0} & \theta_{12}^{0} & \theta_{13}^{0} \\
\theta_{21}^{0} & \theta_{22}^{0} & \theta_{23}^{0} \\
\theta_{31}^{0} & \theta_{32}^{0} & \theta_{33}^{0}
\end{array}\right]=\left[\begin{array}{ccc}
0.2 & 0.21 & 0.19 \\
0.217 & 0.217 & 0.22 \\
0.17 & 0.21 & 0.2
\end{array}\right],} \\
& {\left[\begin{array}{lll}
\theta_{11} & \theta_{12} & \theta_{13} \\
\theta_{21} & \theta_{22} & \theta_{23} \\
\theta_{31} & \theta_{32} & \theta_{33}
\end{array}\right]=\left[\begin{array}{ccc}
0.27 & 0.32 & 0.265 \\
0.317 & 0.372 & 0.325 \\
0.23 & 0.3 & 0.26
\end{array}\right] .}
\end{aligned}
$$

It is easy to check that

$$
\begin{gathered}
A \geq\left\|\varphi^{0}\right\|, \max _{\substack{1 \leq i \leq n \\
1 \leq j \leq m}}\left\{\frac{\theta_{i j}^{0}}{a_{i j}^{m}},\left(1+\frac{a_{i j}^{M}}{a_{i j}^{m}}\right) \theta_{i j}^{0}\right\} \leq \frac{1}{2}, \\
\max _{\substack{1 \leq i \leq n \\
1 \leq j \leq m}}\left\{\frac{\theta_{i j}}{a_{i j}^{m}},\left(1+\frac{a_{i j}^{M}}{a_{i j}^{m}}\right) \theta_{i j}\right\}<1 .
\end{gathered}
$$

Therefore, all the conditions in Theorems 16 and 18 are satisfied. Hence (66) has a $2 \pi$-periodic solution, which is globally exponentially stable (we give numerical simulations in Figures 1, 2, and 3 by taking $x_{11}, x_{12}$, and $x_{13}$ to illustrate our results).

Example 2. In system (66), let $\mathbb{T}=\mathbb{Z}$, then $\mu(t) \equiv 1$, and take

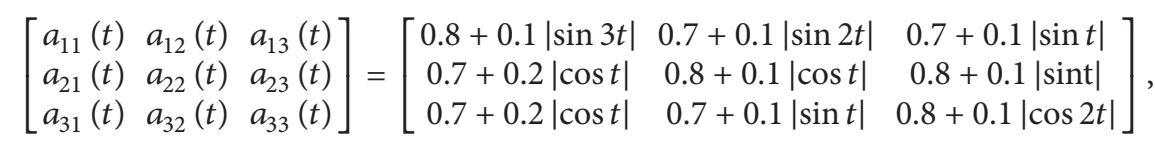

$$
\begin{aligned}
& {\left[\begin{array}{lll}
c_{11}(t) & c_{12}(t) & c_{13}(t) \\
c_{21}(t) & c_{22}(t) & c_{23}(t) \\
c_{31}(t) & c_{32}(t) & c_{33}(t)
\end{array}\right]=\left[\begin{array}{lll}
0.01|\sin 2 t| & 0.02|\sin 3 t| & 0.01|\sin 4 t| \\
0.02|\sin 2 t| & 0.01|\sin 3 t| & 0.01|\cos 4 t| \\
0.01|\sin 2 t| & 0.01|\sin 3 t| & 0.02|\cos 4 t|
\end{array}\right]} \\
& {\left[\begin{array}{lll}
d_{11}(t) & d_{12}(t) & d_{13}(t) \\
d_{21}(t) & d_{22}(t) & d_{23}(t) \\
d_{31}(t) & d_{32}(t) & d_{33}(t)
\end{array}\right]=\left[\begin{array}{lll}
0.01|\cos 2 t| & 0.02|\sin 3 t| & 0.01|\sin 4 t| \\
0.01|\cos 2 t| & 0.01|\cos 3 t| & 0.01|\cos 4 t| \\
0.01|\cos 2 t| & 0.01|\sin 2 t| & 0.02|\cos 4 t|
\end{array}\right]} \\
& {\left[\begin{array}{lll}
I_{11}(t) & I_{12}(t) & I_{13}(t) \\
I_{21}(t) & I_{22}(t) & I_{23}(t) \\
I_{31}(t) & I_{32}(t) & I_{33}(t)
\end{array}\right]=\left[\begin{array}{ccc}
0.08 \sin t+0.03 \cos t & 0.02 \sin t+0.01 \cos 2 t & 0.02 \cos t \\
0.01 \sin t & 0.02 \cos t & 0.06 \cos t \\
0.01 \cos t & 0.01 \sin t+0.02 \sin t & 0.01 \cos t
\end{array}\right],} \\
& {\left[\begin{array}{lll}
\mu_{11}(t) & \mu_{12}(t) & \mu_{13}(t) \\
\mu_{21}(t) & \mu_{22}(t) & \mu_{23}(t) \\
\mu_{31}(t) & \mu_{32}(t) & \mu_{33}(t)
\end{array}\right]=\left[\begin{array}{ccc}
0.02|\sin t| & 0.01|\sin 2 t| & 0.02|\cos t| \\
0.01|\cos 2 t| & 0.01|\sin t| & 0.01|\cos t| \\
0.02|\sin 2 t| & 0.01|\cos t| & 0.02|\sin 2 t|
\end{array}\right] .}
\end{aligned}
$$


Let $r=s=1, A=1 / 2$, and $\delta=0.1$. Obviously, $L=l=2$, $f(0)=2$ and $g(0)=0, K_{i j}^{0}=1 / 4, J_{i j}^{0}=1 / 2$,

$$
\begin{aligned}
& {\left[\begin{array}{ccc}
a_{11}^{M} & a_{12}^{M} & a_{13}^{M} \\
a_{21}^{M} & a_{22}^{M} & a_{23}^{M} \\
a_{31}^{M} & a_{32}^{M} & a_{33}^{M}
\end{array}\right]=\left[\begin{array}{lll}
0.9 & 0.8 & 0.9 \\
0.9 & 0.9 & 0.9 \\
0.9 & 0.8 & 0.9
\end{array}\right],} \\
& {\left[\begin{array}{lll}
a_{11}^{m} & a_{12}^{m} & a_{13}^{m} \\
a_{21}^{m} & a_{22}^{m} & a_{23}^{m} \\
a_{31}^{m} & a_{32}^{m} & a_{33}^{m}
\end{array}\right]=\left[\begin{array}{lll}
0.8 & 0.7 & 0.7 \\
0.7 & 0.8 & 0.8 \\
0.7 & 0.7 & 0.8
\end{array}\right],}
\end{aligned}
$$

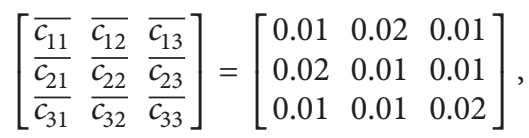

$$
\begin{aligned}
& {\left[\begin{array}{lll}
\overline{d_{11}} & \overline{d_{12}} & \overline{d_{13}} \\
\frac{d_{21}}{d_{31}} & \frac{d_{22}}{d_{32}} & \frac{d_{23}}{d_{33}}
\end{array}\right]=\left[\begin{array}{lll}
0.01 & 0.02 & 0.01 \\
0.01 & 0.01 & 0.01 \\
0.01 & 0.01 & 0.02
\end{array}\right],} \\
& {\left[\begin{array}{lll}
\overline{\frac{I_{11}}{I_{21}}} & \overline{I_{12}} & \overline{I_{13}} \\
\overline{I_{31}} & \frac{I_{22}}{I_{32}} & \frac{I_{33}}{I_{33}}
\end{array}\right]=\left[\begin{array}{lll}
0.11 & 0.03 & 0.02 \\
0.01 & 0.02 & 0.06 \\
0.01 & 0.03 & 0.01
\end{array}\right],} \\
& {\left[\begin{array}{lll}
\mu_{11}^{M} & \mu_{12}^{M} & \mu_{13}^{M} \\
\mu_{21}^{M} & \mu_{22}^{M} & \mu_{23}^{M} \\
\mu_{31}^{M} & \mu_{32}^{M} & \mu_{33}^{M}
\end{array}\right]=\left[\begin{array}{lll}
0.02 & 0.01 & 0.02 \\
0.01 & 0.01 & 0.01 \\
0.02 & 0.01 & 0.02
\end{array}\right] \text {. }}
\end{aligned}
$$

So

$$
\begin{aligned}
& {\left[\begin{array}{lll}
\theta_{11}^{0} & \theta_{12}^{0} & \theta_{13}^{0} \\
\theta_{21}^{0} & \theta_{22}^{0} & \theta_{23}^{0} \\
\theta_{31}^{0} & \theta_{32}^{0} & \theta_{33}^{0}
\end{array}\right]=\left[\begin{array}{lll}
0.128 & 0.158 & 0.118 \\
0.159 & 0.229 & 0.169 \\
0.108 & 0.158 & 0.118
\end{array}\right],} \\
& {\left[\begin{array}{llll}
\theta_{11} & \theta_{12} & \theta_{13} \\
\theta_{21} & \theta_{22} & \theta_{23} \\
\theta_{31} & \theta_{32} & \theta_{33}
\end{array}\right]=\left[\begin{array}{lll}
0.208 & 0.288 & 0.193 \\
0.269 & 0.389 & 0.289 \\
0.133 & 0.268 & 0.193
\end{array}\right] .}
\end{aligned}
$$

It is easy to check that

$$
\begin{gathered}
A \geq\left\|\varphi^{0}\right\|, \max _{\substack{1 \leq i \leq n \\
1 \leq j \leq m}}\left\{\frac{\theta_{i j}^{0}}{a_{i j}^{m}},\left(1+\frac{a_{i j}^{M}}{a_{i j}^{m}}\right) \theta_{i j}^{0}\right\} \leq \frac{1}{2}, \\
\max _{\substack{1 \leq i \leq n \\
1 \leq j \leq m}}\left\{\frac{\theta_{i j}}{a_{i j}^{m}},\left(1+\frac{a_{i j}^{M}}{a_{i j}^{m}}\right) \theta_{i j}\right\}<1 .
\end{gathered}
$$

Therefore, all the conditions in Theorems 16 and 18 are verified. Hence (66) has a $2 \pi$-periodic solution, which is globally exponentially stable(we give numerical simulations in Figures 4, 5, and 6 by taking $x_{11}, x_{12}$, and $x_{13}$ to illustrate our results).

Remark. In [27], authors studied the existence and stability of antiperiodic solutions to impulsive shunting inhibitory cellular neural networks with distributed delays on time scales by using a continuation theorem of coincidence degree

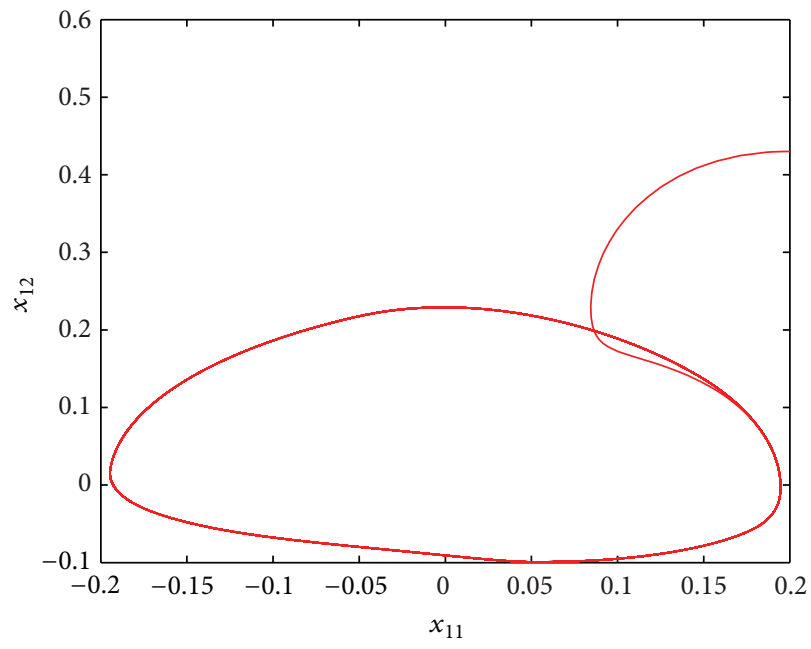

FiguRE 1: Phase response of state variables $x_{11}, x_{12}$ in Example 1.

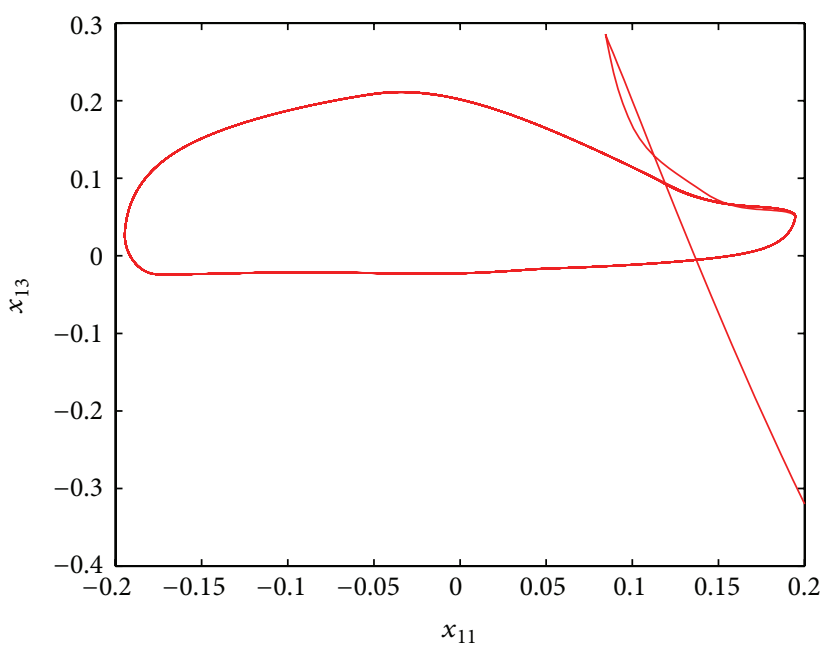

Figure 2: Phase response of state variables $x_{11}$ and $x_{13}$ in Example 1.

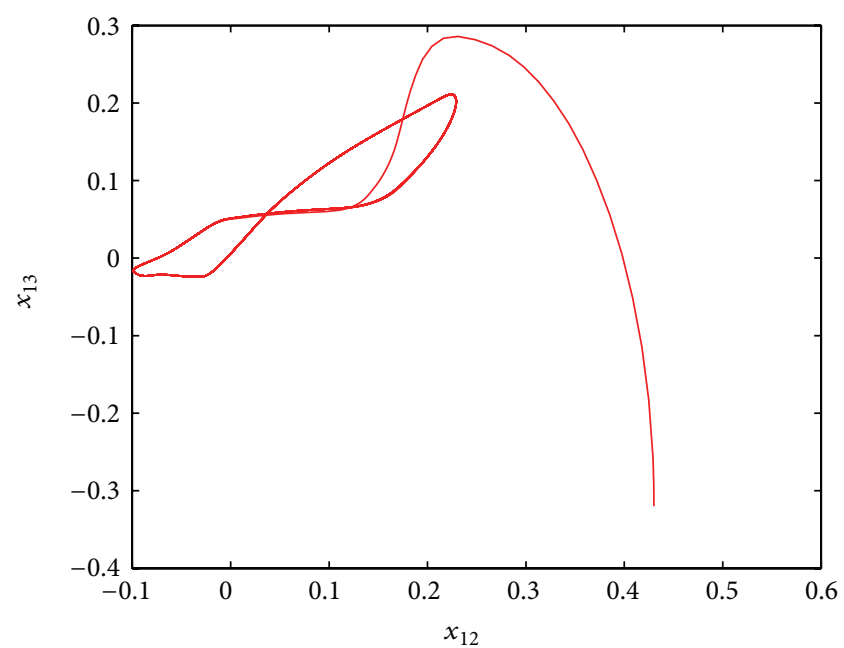

Figure 3: Phase response of state variables $x_{12}$ and $x_{13}$ in Example 1. 


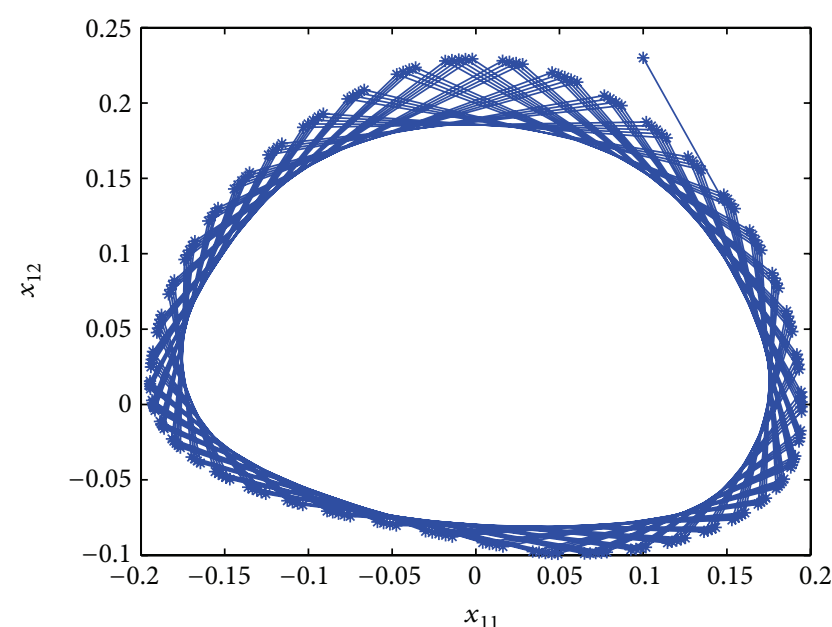

Figure 4: Phase response of state variables $x_{11}, x_{12}$ in Example 2.

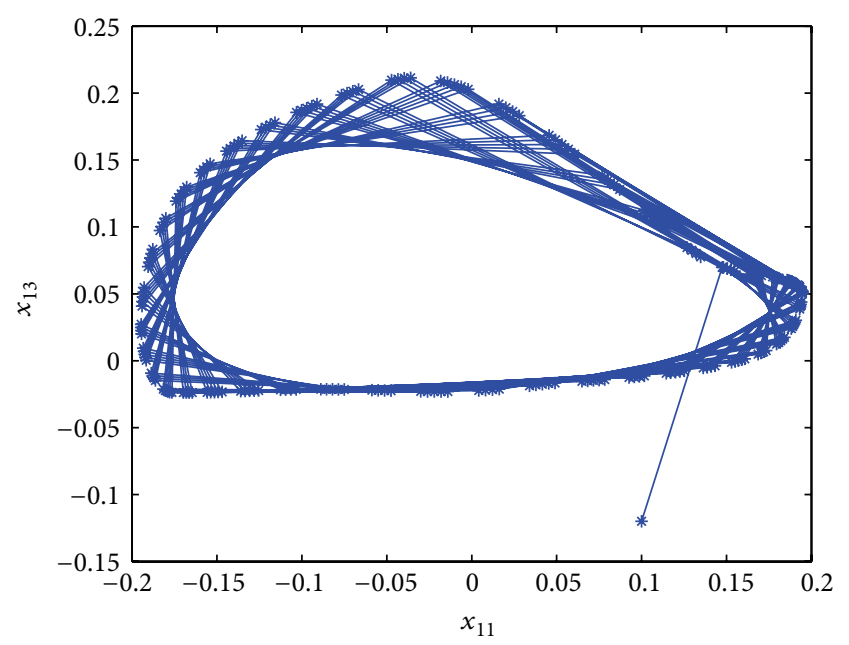

Figure 5: Phase response of state variables $x_{11}, x_{13}$ in Example 2.

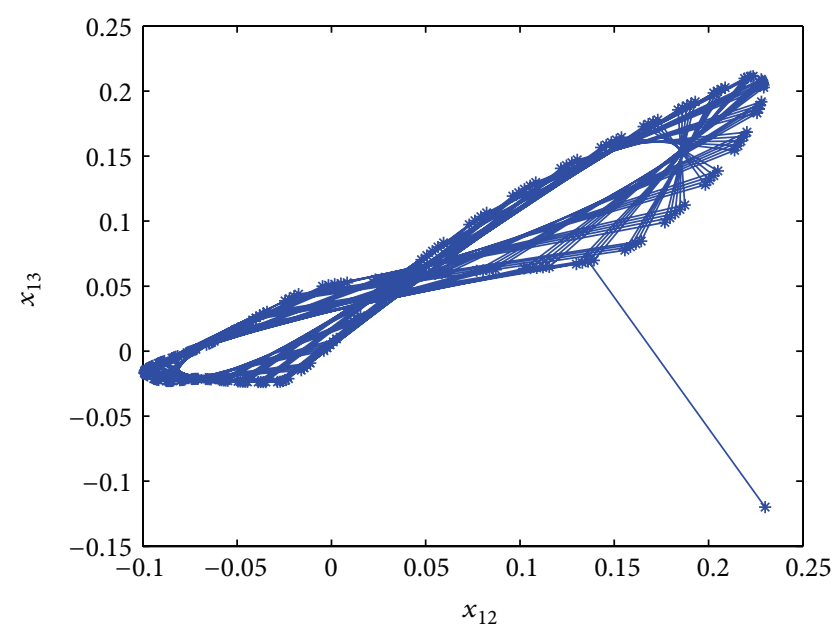

Figure 6: Phase response of state variables $x_{12}, x_{13}$ in Example 2. theory and constructing a suitable Lyapunov functions, and in [28], authors investigated the existence and stability of almost periodic solutions to shunting inhibitory cellular neural networks on time scales by the exponential dichotomy of linear dynamic equations on time scales and constructing a suitable Lyapunov functions. However, our methods used in this paper are different from those used in $[27,28]$; also, the results obtained in $[27,28]$ cannot be applied to our examples here.

\section{Conflict of Interests}

The authors declare that there is no conflict of interests regarding the publication of this paper.

\section{Acknowledgment}

This work is supported by the National Natural Sciences Foundation of People's Republic of China under Grants 11361072 and 11061036.

\section{References}

[1] X. Huang and J. Cao, "Almost periodic solution of shunting inhibitory cellular neural networks with time-varying delay," Physics Letters A, vol. 314, no. 3, pp. 222-231, 2003.

[2] Y. Xia, J. Cao, and Z. Huang, "Existence and exponential stability of almost periodic solution for shunting inhibitory cellular neural networks with impulses," Chaos, Solitons \& Fractals, vol. 34, no. 5, pp. 1599-1607, 2007.

[3] C. Ou, "Almost periodic solutions for shunting inhibitory cellular neural networks," Nonlinear Analysis: Real World Applications, vol. 10, no. 5, pp. 2652-2658, 2009.

[4] X. Yang, "Existence and global exponential stability of periodic solution for Cohen-Grossberg shunting inhibitory cellular neural networks with delays and impulses," Neurocomputing, vol. 72, no. 10-12, pp. 2219-2226, 2009.

[5] Y. Li, C. Liu, and L. Zhu, "Global exponential stability of periodic solution for shunting inhibitory CNNs with delays," Physics Letters A, vol. 337, no. 1-2, pp. 46-54, 2005.

[6] B. Liu, "Stability of shunting inhibitory cellular neural networks with unbounded time-varying delays," Applied Mathematics Letters, vol. 22, no. 1, pp. 1-5, 2009.

[7] B. Liu, "New convergence behavior of solutions to shunting inhibitory cellular neural networks with unbounded delays and time-varying coefficients," Applied Mathematical Modelling, vol. 33, no. 1, pp. 54-60, 2009.

[8] J. H. Park, O. M. Kwon, and S. M. Lee, "LMI optimization approach on stability for delayed neural networks of neutraltype," Applied Mathematics and Computation, vol. 196, no. 1, pp. 236-244, 2008.

[9] J. H. Park, C. H. Park, O. M. Kwon, and S. M. Lee, "A new stability criterion for bidirectional associative memory neural networks of neutral-type," Applied Mathematics and Computation, vol. 199, no. 2, pp. 716-722, 2008.

[10] S. M. Lee, O. M. Kwon, and J. H. Park, "A novel delay-dependent criterion for delayed neural networks of neutral type," Physics Letters A, vol. 374, no. 17-18, pp. 1843-1848, 2010. 
[11] J. H. Park and O. M. Kwon, "Global stability for neural networks of neutral-type with interval time-varying delays," Chaos, Solitons and Fractals, vol. 41, no. 3, pp. 1174-1181, 2009.

[12] J. H. Park and O. M. Kwon, "Further results on state estimation for neural networks of neutral-type with time-varying delay," Applied Mathematics and Computation, vol. 208, no. 1, pp. 6975, 2009.

[13] J. H. Park, "Synchronization of cellular neural networks of neutral type via dynamic feedback controller," Chaos, Solitons and Fractals, vol. 42, no. 3, pp. 1299-1304, 2009.

[14] Y. Li, L. Zhao, and X. Chen, "Existence of periodic solutions for neutral type cellular neural networks with delays," Applied Mathematical Modelling, vol. 36, no. 3, pp. 1173-1183, 2012.

[15] Z. Gui, W. Ge, and X.-S. Yang, "Periodic oscillation for a Hopfield neural networks with neutral delays," Physics Letters A, vol. 364, no. 3-4, pp. 267-273, 2007.

[16] L. Li, Z. Fang, and Y. Yang, "A shunting inhibitory cellular neural network with continuously distributed delays of neutral type," Nonlinear Analysis: Real World Applications, vol. 13, no. 3, pp. 1186-1196, 2012.

[17] P. Balasubramaniam, G. Nagamani, and R. Rakkiyappan, "Passivity analysis for neural networks of neutral type with Markovian jumping parameters and time delay in the leakage term," Communications in Nonlinear Science and Numerical Simulation, vol. 16, no. 11, pp. 4422-4437, 2011.

[18] X. Li and J. Cao, "Delay-dependent stability of neural networks of neutral type with time delay in the leakage term," Nonlinearity, vol. 23, no. 7, pp. 1709-1726, 2010.

[19] B. Liu, "Global exponential stability for BAM neural networks with time-varying delays in the leakage terms," Nonlinear Analysis: Real World Applications, vol. 14, no. 1, pp. 559-566, 2013.

[20] P. Balasubramaniam, M. Kalpana, and R. Rakkiyappan, "Existence and global asymptotic stability of fuzzy cellular neural networks with time delay in the leakage term and unbounded distributed delays," Circuits, Systems, and Signal Processing, vol. 30, no. 6, pp. 1595-1616, 2011.

[21] X. Li, R. Rakkiyappan, and P. Balasubramaniam, "Existence and global stability analysis of equilibrium of fuzzy cellular neural networks with time delay in the leakage term under impulsive perturbations," Journal of the Franklin Institute, vol. 348, no. 2, pp. 135-155, 2011.

[22] S. Lakshmanan, J. H. Park, H. Y. Jung, and P. Balasubramaniam, "Design of state estimator for neural networks with leakage, discrete and distributed delays," Applied Mathematics and Computation, vol. 218, no. 22, pp. 11297-11310, 2012.

[23] X. Li, X. Fu, P. Balasubramaniam, and R. Rakkiyappan, "Existence, uniqueness and stability analysis of recurrent neural networks with time delay in the leakage term under impulsive perturbations," Nonlinear Analysis: Real World Applications, vol. 11, no. 5, pp. 4092-4108, 2010.

[24] K. Gopalsamy, "Leakage delays in BAM," Journal of Mathematical Analysis and Applications, vol. 325, no. 2, pp. 1117-1132, 2007.

[25] C. Li and T. Huang, "On the stability of nonlinear systems with leakage delay," Journal of the Franklin Institute, vol. 346, no. 4, pp. 366-377, 2009.

[26] S. Hilger, "Analysis on measure chains-a unified approach to continuous and discrete calculus," Results in Mathematics, vol. 18, no. 1-2, pp. 18-56, 1990.

[27] Y. Li and J. Shu, "Anti-periodic solutions to impulsive shunting inhibitory cellular neural networks with distributed delays on time scales," Communications in Nonlinear Science and Numerical Simulation, vol. 16, no. 8, pp. 3326-3336, 2011.

[28] Y. Li and C. Wang, "Almost periodic solutions of shunting inhibitory cellular neural networks on time scales," Communications in Nonlinear Science and Numerical Simulation, vol. 17, no. 8, pp. 3258-3266, 2012.

[29] M. Bohner and A. Peterson, Eds., Advances in Dynamic Equations on Time Scales, Birkhäuser, Boston, Mass, USA, 2003.

[30] E. R. Kaufmann and Y. N. Raffoul, "Periodic solutions for a neutral nonlinear dynamical equation on a time scale," Journal of Mathematical Analysis and Applications, vol. 319, no. 1, pp. 315-325, 2006.

[31] J. Zhang, M. Fan, and H. Zhu, "Existence and roughness of exponential dichotomies of linear dynamic equations on time scales," Computers \& Mathematics with Applications, vol. 59, no. 8, pp. 2658-2675, 2010. 


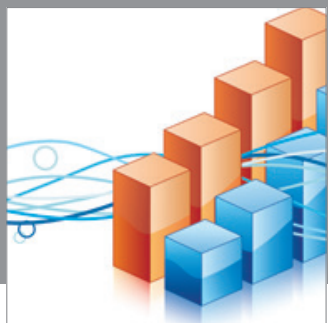

Advances in

Operations Research

mansans

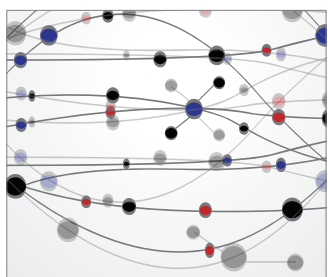

The Scientific World Journal
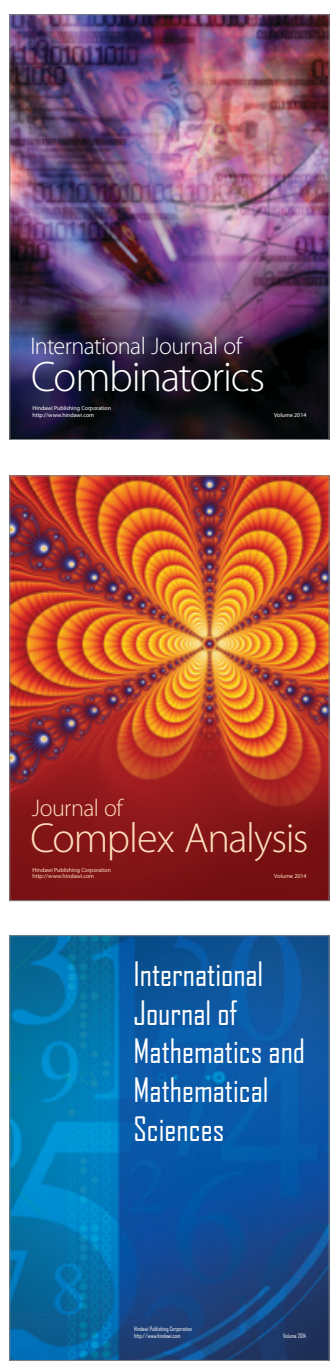
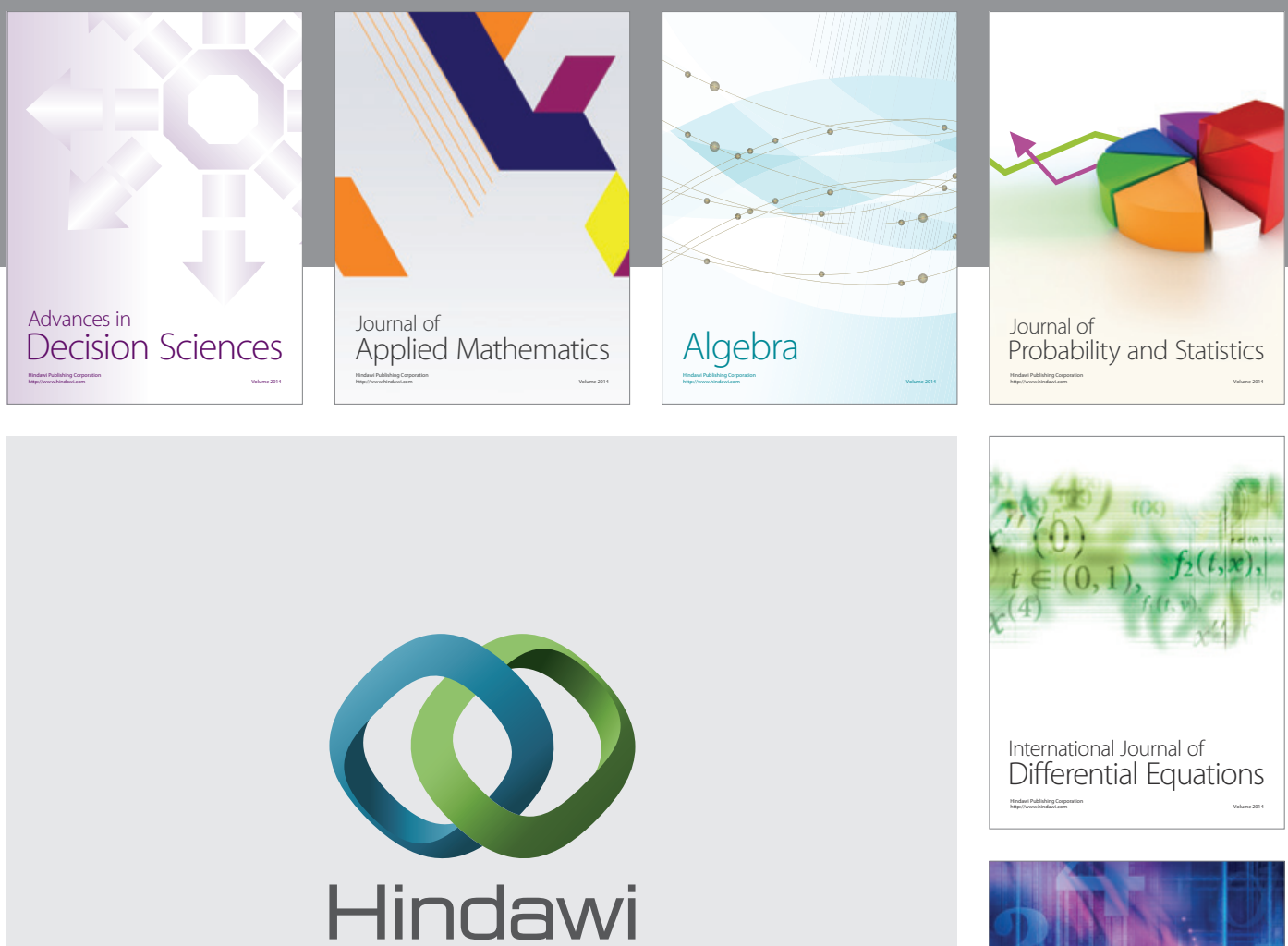

Submit your manuscripts at http://www.hindawi.com
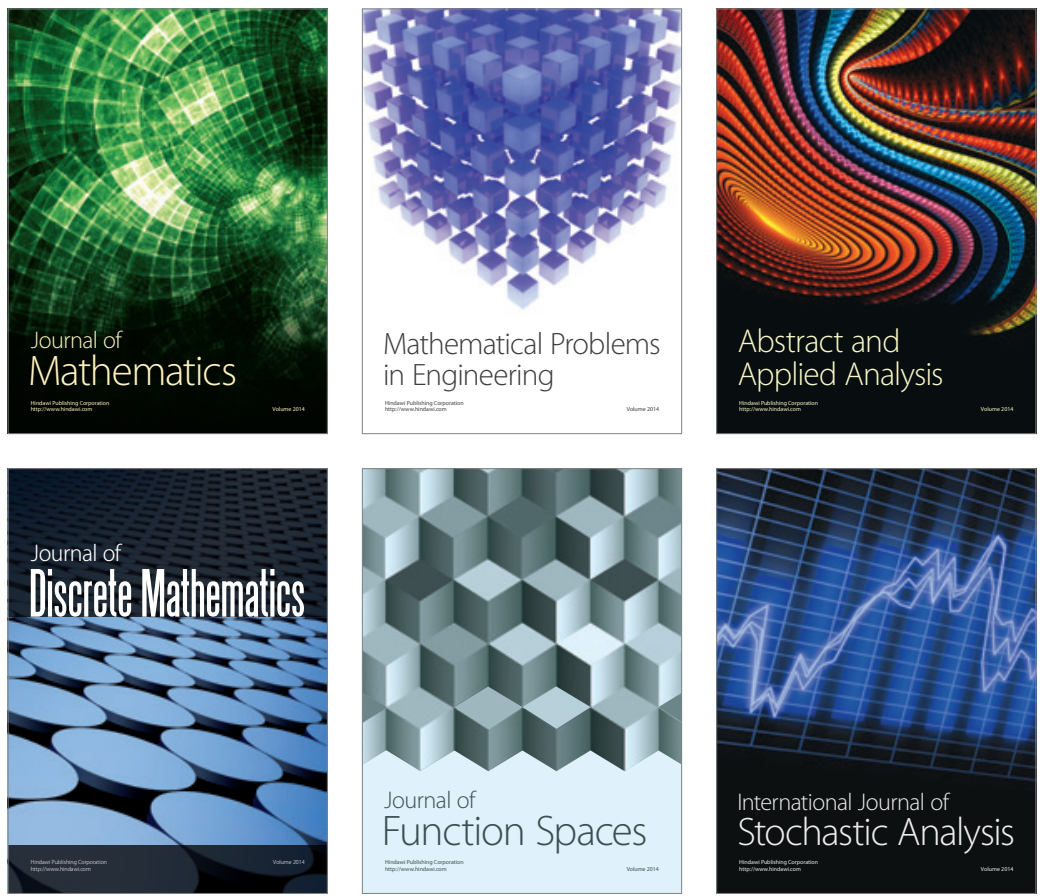

Journal of

Function Spaces

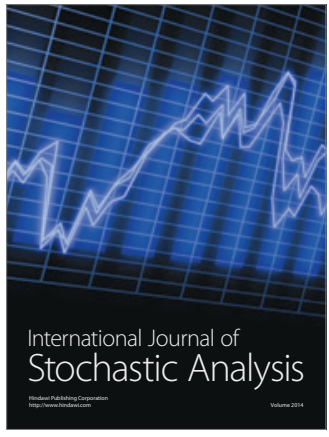

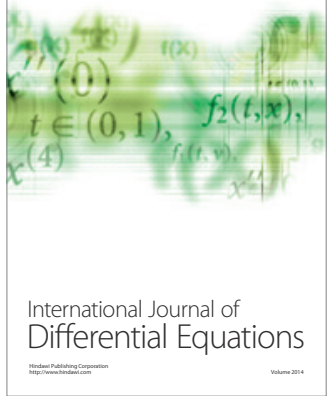
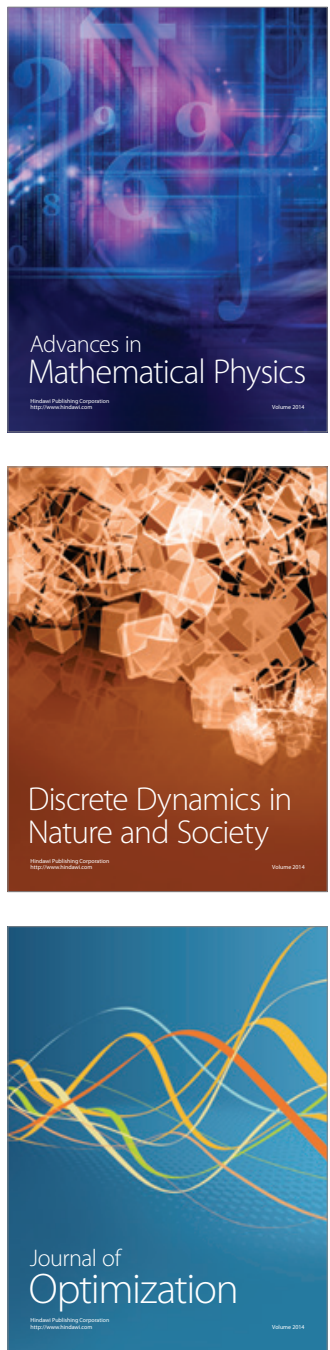